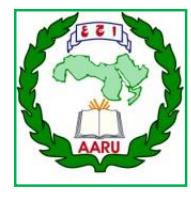

Arab Univ. J. Agric. Sci., Ain Shams Univ., Cairo, Egypt

28(2), 505-520, 2020

Website: http://ajs.journals.ekb.eg

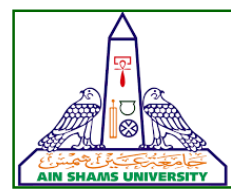

505

\title{
EFFECTIVENESS OF SOME AROMATIC OILS TREATMENTS ON QUALITY CONSERVATION OF PEPPERMINT FRESH CUT HERB UNDER COLD STORAGE
}

\author{
Abdelhamid ${ }^{\star}$ A.N. \\ Horticulture Dept., Fac. of Agric., Ain Shams Univ., P.O. Box 68, Hadayek Shoubra 11241, \\ Cairo, Egypt \\ *Corresponding author: nazmy604@yahoo.com
}

Accepted 4 August, 2020

\section{ABSTRACT}

The effect of lavender, lemongrass and thyme volatile oils as a supplementary cold storage treatments on quality preservation of fresh cut herb of Menthe piperita L., during storage for 15 days at $5^{\circ} \mathrm{C}$ and $7^{\circ} \mathrm{C}$ followed by 5 days at $20^{\circ} \mathrm{C}$, as a marketing condition simulation, was evaluated during 2018 and 2019 seasons. Physical properties, chemical constituents, respiration rate and volatile oil contents were recorded. The results illustrated that discarded herb \%, weight loss \%, and external appearance (scale) were greatly affected by both factors of the study. The great effect in this respect was obtained with storage at $5^{\circ} \mathrm{C}$ than $7^{\circ} \mathrm{C}$ and the effect was also continuous during marketing period at $20^{\circ} \mathrm{C}$. An evident decrease in discarded herb $\%$ and weight loss \% were obtained due to the applied treatments, whereas external appearance values were higher with applied treatments than control during cold storage durations and marketing period. Aromatic oils treatments with $0.50 \%$ of lavender, lemongrass and thyme oils were effective than $0.25 \%$ of the three aromatic oils. However, chemical constituents i.e total chlorophyll, L-ascorbic acid and total phenols of fresh cut herb were scored highly increases by storage at $5^{\circ} \mathrm{C}$ than at $7^{\circ} \mathrm{C}$ storage conditions. Furthermore, The richest chemical constituents mentioned above were obtained with $0.50 \%$ of the three aromatic oils treatments and with superior for marketing durations for 5 days at $20^{\circ} \mathrm{C}$. On contrary, total acidity of fresh cut herb recorded the minimum values with applied treatments than control (except the treatment of $0.25 \%$ thyme oil) during all cold storage durations and marketing pe- riod. On the reverse, electrolyte leakage and respiration rate values were controlled and minimized with the applied aromatic oils treatments under $5^{\circ} \mathrm{C}$ or $7^{\circ} \mathrm{C}$ cold storage conditions and followed by 5 days at $20^{\circ} \mathrm{C}$ as a marketing period. The decreases of both electrolyte leakage and respiration rate of fresh cut herb either with cold storage or due to the applied treatments are considered a good indicators to increase storage longevity and high quality. Meanwhile, volatile oil was greatly affected by both cold storage conditions and supplementary refrigeration treatments with lavender, lemongrass and thyme aromatic oils. Conclusively, it could be concluded that the peppermint fresh cut herb can be treated with lavender, lemongrass and thyme aromatic oils before storage at $5^{\circ} \mathrm{C}$ for 15 days, handling and marketing under $20^{\circ} \mathrm{C}$ to conserve the quality of the herb.

Keywords: Peppermint, fresh cut herb, aromatic oil, Lavender, Lemongrass and Thyme, cold storage, supplementary refrigeration, respiration rate

\section{INTRODUCTION}

Peppermint (Mentha piperita L.) is an important species and one of the most well-known herb of the Lamiacene family which having 25 species (Karkanis et al 2018). Additionally, spearmint (Mentha spicata L.) also is a perennial crop cultivated for its aromatic oils that are applied mostly in flavoring gum and dental hygiene products. Regarding to peppermint plant, it is a hybrid between watermint (Mentha aquatic L.) and spearmint (Mentha spicata L.) had many advantages such as health promoting and recuperating properties, the trademark reviving fragrance, makes mint one of the most attractive 
herbs for use in different plates of mixed greens just as flower and trimming in foods. However the major contributor to world peppermint production is Asia with $92.23 \%$ of total production and is followed by Americas which contributes $7.61 \%$. Different species of mentha had many health benefits, nutritional supplement in livestock feed (Hosoda et al 2005), food and peppermint freshener. So, since ancient times mint species are being consumed without any side effect and they are generally considered safe for human and animal consumption due to they are rich in polyphenols and flavones in different form. The main components are important in human health as a source for vitamins and as antioxidant (Maisuthisakul et al 2007).

Medicinal and aromatic plants essential oils represent an alternative to pesticide application in the production processes of agriculture food and commodities preservation during storage and handling (Camele et al 2010 and Plaza et al 2004). Since, various natural aromatic oils have been used to control plant diseases and prevent product losses due to microbial effect and consequently improved external appearance and reduced herb decay \% (Heidarpour et al 2013 and Ghanbari et al 2016). It is well known that plant-based products such aromatic oil extracted from different aromatic plants are safe substances and good candidate for control of storage conditions as reported by Isman, 2000 \& Isman and Machial 2006. The demand for food product free of pesticide and chemicals is growing. It has been demonstrated that postharvest deteriorations and preservation quality of stored commodities can be controlled by plant essential oils or plant extracts (Plaza et al 2004). Plant essential oils have antifungal and antibacterial effects depending on plant species and applied oil concentrations (Arras \& Usai 2001, Camele et al 2010, Combrinck et al 2011, Lalitha et al 2011 \& Mari et al 2016) causing evident decrease in weight loss $\%$, decay $\%$ and an increase in external appearances.

However, many studies indicated the efficiency of essential oils and plant extract of many aromatic plants on fungal and bacterial diseases and consequently increase storability of different commodities (Lee et al 2008, Lalitha et al 2011). In this respect, lemongrass (Cymbopogon citratus L.) oil was reported by Hyun et al 2015, to decrease fungal activity of several plant pathogens and a reduction in disease incidence. However, weight loss, loss of fruit succulence and showed acceptable appearance during cold storage at market shelf conditions of avocado fruits were reported by Mpho et al 2013. In addition, thyme (Thymus cupitates L.) oil has been used to control many plant diseases of several fruits (Abd-alla et al 2011). It was effective in preserving the losses of vitamin $\mathrm{C}$ and maintaining quality of the orange fruits (Fatemi et al 2011). Also, bergamot (Citrus bergamia) oil minimized fruit decay, weight loss $\%$ and delayed the change in fruit softening, total acidity, T.S.S\%, vitamin C, anthocyamin pigment content and respiration rate of crimson seedless grapes (Abd-Elwahab et al 2014).

Similar trend of results on the great effect as aromatic oils as a supplementary refrigeration treatments were also found by peppermint oil on orange fruits (Fatemi et al 2011) and plum fruits (Aminifard and Mohammadi, 2013), rosemary oil on many commodities (Almela et al 2006), lavender oil, dill oil and coriander oil (Serban et al 2011). It could be discussed as a general view that, the role of essential oils act as antibiotic activity, allelopathy, attractants, feed in deterrents and phytoalexin (Mahanta, 2007).

The aim of this research was to evaluate the effect of aromatic oils of lavender, lemongrass and thyme plants as a supplementary refrigeration treatments on improving storability of peppermint fresh cut herb during storage at 5 or $7^{\circ} \mathrm{C} \pm 1$ and $95 \% \mathrm{RH}$ for 15 days followed by 5 days at $20^{\circ} \mathrm{C} \pm 2$ as a marketing simulation conditions, during 2018 and 2019 seasons.

\section{MATERIALS AND METHODS}

The present work was conducted during 2018 and 2019 seasons in Hort. Dept. Fac.of Agric., Ain Shams Univ., on the fresh cut herb of (Mentha piperita L.) Baladi cv. The aerial part of plant (main aerial stem, branches and leaves) were cut on the first of May in both seasons. The samples of herbs plants were obtained from a private farm located in Wadi El- Natrun region, Behira Governorate .After clean up and dipping in cold water for 5 minutes, the herb was rinsed in percidine bio-fungicide $(25 \mathrm{mg} / \mathrm{L})$ for 5 minutes (Yousefizad et al 2015), then subjected to air drying , the percidine - treated herb was dipped in distilled water and air dried.The cleaned herb of plant was adapted to $10 \mathrm{~cm}$ - branches length and bundled in $50 \mathrm{gm}$. for each bundle and subjected to pre - storage treatments as dipping for 5 minutes as follows :-

1. Control washed in distilled water

2. Lavender oil at $0.25 \%$

3. Lavender oil at $0.50 \%$

4. Lemongrass oil at $0.25 \%$

5. Lemongrass oil at $0.50 \%$

6. Thyme oil at $0.25 \%$ 
7. Thyme oil at $0.50 \%$

After the treatments, the bundles were air dried, packed in proliferated cellophane bags and held in foam dishes. A completely randomized block design with 4 replicates was used, each replicate contained from 10 herb foam dishes ( 7 treatments $\times 3$ replicates $\times 30$ dishes $=630$ dishes for each temperatures storage. All dishes were stored at 5 and $7 \pm 1$ ${ }^{\circ} \mathrm{C}$ for 15 days (relative humidity $95 \%$ ), followed by 5 days at $20 \pm 2{ }^{\circ} \mathrm{C}$ as a marketing condition simulation. Initial sample from each temperature storage was taken for different analysis.After that, samples were pull from cold storage at 5,10 and 15 days and them subjected to different analysis whereas a part of sample (3 dishes) for each replicate was held at $20 \pm 2 \stackrel{\circ}{\circ}$ for 5 days as a marketing condition .

The following parameters were evaluated:-

\section{Physical properties}

- Discarded Herb \%: The grams of decayed herbs was periodically recorded and expressed as a percentage from the total number.

- Weight loss \%: Herbs were weighed at the beginning of the experiment just after treatments application and every 5-day interval during the storage period. Weight loss was expressed as the percentage loss of the initial total weight.

- External appearance (scale): was rated subjectively into one of five categories: 5 (excellent), 4 ( very good) , 3 (good), 2 (fair), and 1 (poor), by an informal panel of five people (Erkan et al 2005)

\section{Chemical analysis}

- Total chlorophyll (as $\mathrm{mg} / \mathrm{g}$ fresh herb): was determined according to Nagata and Yamashta 1992

- Total acidity (as g Citric acid/100 g fresh herb) was determined according to A.O.A.C. 2005.

- L-ascorbic acid content ( $\mathrm{mg} / \mathrm{g}$ fresh herb) was determined following the methods by A.O.A.C. 2005
- Electrolyte leakage (\% EC leakage): Ten grams disks of the herb tissues were placed in a $100-\mathrm{ml}$ glass beaker containing $30 \mathrm{ml}$ of deionized water and magnetic stirred for $15 \mathrm{~min}$. Electrical conductivity (EC) of the stirrered solution was measured using electrical conductivity meter. Stirrered solution of each beaker was then replaced by equal volume $(30 \mathrm{ml})$ of deionized water for homogenizing the disks in a blender, and the aliquot was then used for measuring EC level as previously described. Percentage of solute leakage was then calculated as EC leakage using the method described by Mirdehghan et al 2007

- Total phenoles (as $\mathbf{~ m g / 1 0 0 ~ g ~ d r y ~ h e r b ) : ~ w a s ~}$ measured according to Singleton and Rossi, 1965.

- Total flavones (as mg/100 g dry herb): was determined according to Dewanto et al 2002.

\section{Respiration rate $(\mathrm{mg} \mathrm{CO} / \mathrm{kg}$ fresh herb $/ \mathrm{hr}$.):}

Carbon dioxide produced by herb was determined after $10 \mathrm{hrs}$ finished from treatments and then every 5 days during storage until experiment termination. The air-flow was passed through concentrated $\mathrm{NaOH}$, to insure that air-flow is $\mathrm{CO}_{2}$ free, before passing into 1-liter jar container (herb ambient) $50 \mathrm{~g} / \mathrm{jar}$ ( one dish ) was considered as one replicate. The out-coming air-flow was then passed into $100 \mathrm{ml} \mathrm{NaOH}$ of $0.1 \mathrm{~N}$ for $1 \mathrm{~h}$. Such solution was then titrated against $0.1 \mathrm{~N} \mathrm{HCl}$ and $\mathrm{CO}_{2}$ levels produced by the fresh herb was then calculated as (mg $\mathrm{CO}_{2} / \mathrm{kg}$ fresh herb/hr), according to A.O.A.C. 2005

\section{Volatile oil content (\%)}

In both seasons, the essential oil was extracted by water distillation method according to Novak et al (2002). The amount of obtained volatile oil was measured and oil percentages (\%) were measured according to Charles and Simon (1990).

\section{Statistical analysis}

Data obtained were subjected to analysis of variance (ANOVA) using MSTAT-C software (MSTAT, Michigan University East Lansing). Duncan multiple rang test (LSR) was performed to determine any significant difference among various treatments. $p<0.05$ was selected as decision for significant differences, according to Steel et al (1997). 


\section{RESULTS AND DISCUSSION}

\section{Physical properties}

\section{1-1. Discarded herb \%}

A great effect of applied treatments and cold storage process on discarded herb \% of mentha (Table 1) was recorded in both studied seasons. However, untreated fresh herb recorded the highest values of discarded herb \% followed by the thyme oil treatment at $0.25 \%$ after 15 days of cold storage at $5^{\circ} \mathrm{C}$. The lowest values of discarded herb\% were recorded by lavender and lemongrass oils both at $0.50 \%$ in the two seasons. Moreover, during mar- keting duration condition $\left(20^{\circ} \mathrm{C}\right)$ all applied treatments of aromatic oils were effective in reducing discarded herb \% than control, with the exception of $0.25 \%$ of thyme oil treatment. Generally, storage at $7^{\circ} \mathrm{C}$ exhibited higher values of discarded herb \% than those at $5^{\circ} \mathrm{C}$, in both studied seasons Lavender and lemongrass oils treatments both at $0.50 \%$ recorded during storage on $5^{\circ} \mathrm{C}$ the least discarded herb $\%$. Marketing duration for 5 days at $20^{\circ} \mathrm{C}$ after cold storage process at $7^{\circ} \mathrm{C}$ exhibited higher values of discarded herb than that recorded at $5^{\circ} \mathrm{C}$. The obtained data showed, in general, that the effect of aromatic oils treatments was more pronounced on mentha fresh cut herb with storage at $5^{\circ} \mathrm{C}$ than at $7^{\circ} \mathrm{C}$.

Table 1. Effect of some aromatic oils treatments on discarded herb $\%$ of mentha fresh cut herb stored at $5^{\circ}$ and $7^{\circ} \mathrm{C} \pm 1$ and $95 \% \mathrm{RH}$, followed by 5 days at $20 \pm 2{ }^{\circ} \mathrm{C}$, during 2018 and 2019 seasons

\begin{tabular}{|c|c|c|c|c|c|c|c|c|}
\hline \multirow{4}{*}{ Treatments } & \multicolumn{8}{|c|}{ Temperatures storage } \\
\hline & \multicolumn{3}{|c|}{$\left(5 \pm 1^{\circ} \mathrm{C}\right)$} & \multirow{3}{*}{$\begin{array}{c}5 \text { days } \\
\text { at } \\
\left(20 \pm 1^{\circ} \mathrm{C}\right)\end{array}$} & \multicolumn{3}{|c|}{$\left(7 \pm 1^{\circ} \mathrm{C}\right)$} & \multirow{3}{*}{$\begin{array}{c}5 \text { days } \\
\text { at } \\
\left(20 \pm 1^{\circ} \mathrm{C}\right)\end{array}$} \\
\hline & \multicolumn{3}{|c|}{ Storage durations (days) } & & \multicolumn{3}{|c|}{ Storage durations (days) } & \\
\hline & 5 & 10 & 15 & & 5 & 10 & 15 & \\
\hline \multicolumn{9}{|c|}{2018 Season } \\
\hline Control & $3.91 \mathrm{a}$ & $7.27 \mathrm{a}$ & $12.65 \mathrm{a}$ & $20.66 \mathrm{a}$ & $5.26 \mathrm{a}$ & $17.67 \mathrm{a}$ & -- & -- \\
\hline Lavender oil $0.25 \%$ & $0.0 \mathrm{c}$ & $4.13 b$ & $6.76 \mathrm{~b}$ & $11.33 \mathrm{~b}$ & $2.71 \mathrm{c}$ & $6.21 \mathrm{c}$ & $12.43 b$ & $23.37 \mathrm{a}$ \\
\hline Lavender oil $0.50 \%$ & $0.0 \mathrm{c}$ & $0.0 \mathrm{~d}$ & $0.0 \mathrm{~d}$ & $4.11 \mathrm{de}$ & $0.0 \mathrm{~d}$ & $0.0 \mathrm{e}$ & $5.22 \mathrm{~d}$ & $7.40 \mathrm{c}$ \\
\hline Lemongrass oil $0.25 \%$ & $0.0 \mathrm{c}$ & $2.55 c$ & $3.83 c$ & $9.52 \mathrm{bc}$ & $0.0 \mathrm{~d}$ & $3.98 d$ & $6.56 \mathrm{c}$ & $13.82 b$ \\
\hline Lemongrass oil $0.50 \%$ & $0.0 \mathrm{c}$ & $0.0 \mathrm{~d}$ & $1.74 \mathrm{~d}$ & $3.13 \mathrm{e}$ & $0.0 \mathrm{~d}$ & $0.0 \mathrm{e}$ & $4.91 \mathrm{~d}$ & $7.57 \mathrm{c}$ \\
\hline Thyme oil $0.25 \%$ & $2.72 b$ & $6.23 \mathrm{a}$ & $11.79 \mathrm{a}$ & $17.87 \mathrm{a}$ & $3.95 b$ & $10.88 b$ & $22.57 \mathrm{a}$ & -- \\
\hline Thyme oil $0.50 \%$ & $0.0 \mathrm{c}$ & $0.0 \mathrm{~d}$ & $2.87 \mathrm{~cd}$ & $6.64 \mathrm{~cd}$ & $0.0 \mathrm{~d}$ & $3.17 \mathrm{~d}$ & $6.45 \mathrm{c}$ & $15.45 b$ \\
\hline \multicolumn{9}{|c|}{2019 Season } \\
\hline Control & $3.64 \mathrm{a}$ & $9.46 \mathrm{a}$ & $15.55 \mathrm{a}$ & $27.75 \mathrm{a}$ & $2.74 \mathrm{bc}$ & $12.98 \mathrm{a}$ & $23.02 \mathrm{a}$ & - \\
\hline Lavender oil $0.25 \%$ & $1.88 \mathrm{bc}$ & $3.61 \mathrm{~b}$ & $9.39 \mathrm{~b}$ & $14.79 \mathrm{c}$ & $2.78 a b$ & $5.83 \mathrm{~b}$ & $15.24 b$ & $19.71 \mathrm{a}$ \\
\hline Lavender oil $0.50 \%$ & $0.0 \mathrm{c}$ & $0.0 \mathrm{c}$ & $2.61 \mathrm{de}$ & 6. 32 ef & $0.0 d$ & $0.0 \mathrm{~d}$ & $5.56 \mathrm{de}$ & $8.68 \mathrm{c}$ \\
\hline Lemongrass oil $0.25 \%$ & $0.0 \mathrm{c}$ & $3.46 \mathrm{bc}$ & $6.22 \mathrm{c}$ & $11.87 \mathrm{~cd}$ & $0.0 \mathrm{~d}$ & 3. $97 \mathrm{bc}$ & $9.97 \mathrm{c}$ & $13.98 \mathrm{~b}$ \\
\hline Lemongrass oil $0.50 \%$ & $0.0 \mathrm{c}$ & $0.0 \mathrm{c}$ & $0.0 \mathrm{e}$ & $5.51 \mathrm{f}$ & $0.0 \mathrm{~d}$ & $0.0 \mathrm{~d}$ & $5.11 \mathrm{e}$ & $6.74 \mathrm{~d}$ \\
\hline Thyme oil $0.25 \%$ & $2.35 b$ & $8.14 \mathrm{a}$ & $15.24 \mathrm{a}$ & $21.38 b$ & $3.35 \mathrm{a}$ & $11.74 \mathrm{a}$ & $24.09 \mathrm{a}$ & -- \\
\hline Thyme oil $0.50 \%$ & $0.0 \mathrm{c}$ & $1.85 \mathrm{c}$ & $4.95 \mathrm{~cd}$ & $9.17 \mathrm{de}$ & $2.21 \mathrm{c}$ & $3.22 \mathrm{~cd}$ & $7.86 \mathrm{~cd}$ & $13.57 b$ \\
\hline
\end{tabular}

Values followed by the same letter (s) are not significantly different at $5 \%$ level

The treatment was terminated due to discarded herb \% reached about $20 \%$. 
Various natural aromatic oils have been used to control plant diseases and prevent product losses due to microbial effect and consequently improved external appearances and reduced herb decay \% (Ghanbari et al 2016 Heidarpour et al 2013).

\section{1-2 Weight loss $\%$}

It is clear from data in Table (2) that weight less $\%$ of peppermint fresh cut herb was greatly affected by aromatic oils treatments and cold storage conditions. However, the lowest values of weight loss \% after 15 days at $5^{\circ} \mathrm{C}$ (5.80 and $\left.5.12 \%\right)$ were recorded by lavender and lemongrass oils treatments both at $0.50 \%$ in first seasons of study. On the contrast, thyme and lavender oils treatments, both at $0.25 \%$ exhibited higher values of weight loss $\%$ than control. The same trend of results was also obtained during marketing at $20^{\circ} \mathrm{C}$ for 5 days. Data recorded at $7^{\circ} \mathrm{C}$ storage declared that the weight loss values were higher than those at $5^{\circ} \mathrm{C}$ regardless of the applied treatments. After 15 days of cold storage at $7^{\circ} \mathrm{C}$, the minimum values and favorable values of weight loss $\%$ were recorded by lavender and lemongrass aromatic oils treatments both at $0.50 \%$, whereas the highest value was recorded with $0.25 \%$ thyme oil treatment.

The great effect to essential oils as a supplementary refrigeration treatments could be attributed to that they are made up of many different volatile components varied between species (Mishra and Dubey, 1994). They made edible films and coating upon the storage commodities helping in preservation because they provide a partial barrier to moisture and $\mathrm{CO}_{2}$ (respiration rate) and avoiding chemical components from losses (Olivas and BarbosaCanovas, 2005).

Table 2. Effect of some aromatic oils treatments on weight loss $\%$ of mentha fresh cut herb stored at $5^{\circ}$ and $7^{\circ} \mathrm{C} \pm 1$ and $95 \% \mathrm{RH}$, followed by 5 days at $20 \pm 2^{\circ} \mathrm{C}$, during 2018 and 2019 seasons .

\begin{tabular}{|c|c|c|c|c|c|c|c|c|}
\hline \multirow{4}{*}{ Treatments } & \multicolumn{8}{|c|}{ Temperatures storage } \\
\hline & \multicolumn{3}{|c|}{$\left(5 \pm 1^{\circ} \mathrm{C}\right)$} & \multirow{3}{*}{\begin{tabular}{|c|}
5 days \\
at \\
$\left(20 \pm 1^{\circ} \mathrm{C}\right)$
\end{tabular}} & \multicolumn{3}{|c|}{$\left(7 \pm 1^{\circ} \mathrm{C}\right)$} & \multirow{3}{*}{$\begin{array}{c}5 \text { days } \\
\text { at } \\
\left(20 \pm 1^{\circ} \mathrm{C}\right)\end{array}$} \\
\hline & \multicolumn{3}{|c|}{ Storage durations (days) } & & \multicolumn{3}{|c|}{ Storage durations (days) } & \\
\hline & 5 & 10 & 15 & & 5 & 10 & 15 & \\
\hline \multicolumn{9}{|c|}{2018 Season } \\
\hline Control & $4.45 \mathrm{a}$ & $5.75 \mathrm{~b}$ & $8.66 a b$ & $15.27 \mathrm{a}$ & $4.65 \mathrm{a}$ & $8.96 \mathrm{a}$ & -- & -- \\
\hline Lavender oil $0.25 \%$ & $3.36 \mathrm{~b}$ & $6.72 \mathrm{a}$ & $8.77 a b$ & $13.11 b$ & $4.54 \mathrm{a}$ & $8.30 \mathrm{a}$ & $11.45 \mathrm{a}$ & $18.20 \mathrm{a}$ \\
\hline Lavender oil $0.50 \%$ & $1.88 \mathrm{~d}$ & $4.13 \mathrm{~d}$ & $5.80 \mathrm{~d}$ & $10.22 d$ & $3.07 \mathrm{c}$ & $5.31 \mathrm{c}$ & $7.71 \mathrm{c}$ & $12.20 \mathrm{c}$ \\
\hline Lemongrass oil $0.25 \%$ & $3.28 \mathrm{~b}$ & $5.38 \mathrm{bc}$ & $7.97 \mathrm{~b}$ & $13.53 b$ & $3.67 b$ & $7.11 \mathrm{~b}$ & $10.24 b$ & $15.76 \mathrm{~b}$ \\
\hline Lemongrass oil $0.50 \%$ & $2.26 \mathrm{~cd}$ & $4.58 \mathrm{~cd}$ & $5.12 d$ & $9.11 \mathrm{~d}$ & $3.11 \mathrm{c}$ & $5.12 \mathrm{c}$ & $7.30 \mathrm{c}$ & $13.97 \mathrm{c}$ \\
\hline Thyme oil $0.25 \%$ & $3.47 b$ & $6.83 \mathrm{a}$ & $9.24 \mathrm{a}$ & $16.20 \mathrm{a}$ & $4.71 \mathrm{a}$ & $9.20 \mathrm{a}$ & $12.74 \mathrm{a}$ & -- \\
\hline Thyme oil $0.50 \%$ & $2.73 \mathrm{c}$ & $5.89 a b$ & $6.80 \mathrm{c}$ & $11.20 \mathrm{c}$ & $3.34 \mathrm{c}$ & $6.71 \mathrm{~b}$ & $10.07 b$ & $1.21 \mathrm{~b}$ \\
\hline \multicolumn{9}{|c|}{2019 Season } \\
\hline Control & $3.91 \mathrm{a}$ & $5.49 \mathrm{~b}$ & $7.16 \mathrm{~b}$ & $19.84 \mathrm{a}$ & $4.16 \mathrm{a}$ & $6.87 a b$ & $9.43 \mathrm{a}$ & -- \\
\hline Lavender oil $0.25 \%$ & $3.27 a b$ & $5.69 a b$ & $6.88 \mathrm{~b}$ & $12.72 \mathrm{c}$ & $3.69 a b$ & $5.89 \mathrm{bc}$ & $8.33 a b$ & $\begin{array}{l}16.00 \\
a\end{array}$ \\
\hline Lavender oil $0.50 \%$ & $1.72 \mathrm{c}$ & $3.16 \mathrm{de}$ & $3.16 \mathrm{~d}$ & $8.85 \mathrm{e}$ & $2.19 \mathrm{~d}$ & $4.30 \mathrm{de}$ & $4.85 d$ & $10.89 d$ \\
\hline Lemongrass oil $0.25 \%$ & $3.04 \mathrm{bc}$ & $4.72 \mathrm{bc}$ & $5.36 \mathrm{c}$ & $11.93 \mathrm{c}$ & $3.49 \mathrm{~b}$ & $5.01 \mathrm{~cd}$ & $7.68 \mathrm{bc}$ & $13.66 \mathrm{~b}$ \\
\hline Lemongrass oil $0.50 \%$ & $1.91 \mathrm{c}$ & $2.27 \mathrm{e}$ & $3.45 d$ & $8.44 \mathrm{e}$ & $2.88 \mathrm{~cd}$ & $3.49 \mathrm{e}$ & $5.32 \mathrm{~d}$ & $10.24 \mathrm{~d}$ \\
\hline Thyme oil $0.25 \%$ & $3.42 a b$ & $6.61 \mathrm{a}$ & $9.17 \mathrm{a}$ & $13.91 \mathrm{~b}$ & $4.07 \mathrm{a}$ & $7.77 \mathrm{a}$ & $9.21 \mathrm{a}$ & -- \\
\hline Thyme oil $0.50 \%$ & $2.10 \mathrm{c}$ & $3.94 \mathrm{~cd}$ & $4.09 \mathrm{~d}$ & $10.81 d$ & $2.55 \mathrm{c}$ & $4.24 \mathrm{de}$ & $6.90 \mathrm{c}$ & $12.77 \mathrm{c}$ \\
\hline
\end{tabular}

Values followed by the same letter (s) are not significantly different at $5 \%$ level

The treatment was terminated due to discarded herb \% reached about $20 \%$. 
It has been demonstrated that postharvest deterioration and preservation quality of stored commidities can be controlled by plant essential oils or plant extracts (Plaza et al 2004. Plant essential oils have antifungal and antibacterial effects depending on plant species and applied oil concentrations (Arras and Usai 2001 \& Camele et al 2010 \& Combrinck et al 2011 \& Lalitha et al 2011 \& Mari et al 2016) caused an evident decrease in weight loss \%, decay $\%$ and an increase in external appearances.

\section{1-3 External appearance (scale)}

Table (3) data showed that, external appearance of mentha fresh cut herb was greatly improved due to aromatic oils treatments and cold storage ambient. It is clear that, external appearance was decreased with the long cold storage durations either at 5 or $7^{\circ} \mathrm{C}$, regardless of the applied treatments. However, $5^{\circ} \mathrm{C}$ storage condition was more effective than $7^{\circ} \mathrm{C}$ and the effect was continuous during marketing durations for $5^{\circ} \mathrm{C}$ at $20^{\circ} \mathrm{C}$. Lavender oil, lemongrass oil and thyme oil all at $0.50 \%$ treatments recorded the highest values of external appearance of fresh cut herb under 5 or $7^{\circ} \mathrm{C}$ cold storage. Furthermore, it could be concluded from the obtained data that volatile aromatic oils could be used as a safe health and environmental treatments on edible commodity such as fresh cut herb without health problems. The effect of these aromatic oils was hastened with cold storage process during handling and marketing of commodities under the ideal conditions $\left(20^{\circ} \mathrm{C}\right)$ for fresh cut herb.

It is well known that plant-based products such aromatic oil extracted from different aromatic plants are safe substances and good candidate for control of storage conditions as reported by Isman, 2000 \& Isman and Machial 2006. The demand for food product free of pesticide and chemicals is growing.

Table 3. Effect of some aromatic oils treatments on external appearance herb (scale) of mentha fresh cut herb stored at $5^{\circ}$ and $7{ }^{\circ} \mathrm{C} \pm 1$ and $95 \% \mathrm{RH}$, followed by 5 days at $20 \pm 2{ }^{\circ} \mathrm{C}$, during 2018 and 2019 seasons

\begin{tabular}{|c|c|c|c|c|c|c|c|c|}
\hline \multirow{4}{*}{ Treatments } & \multicolumn{8}{|c|}{ Temperatures storage } \\
\hline & \multirow{2}{*}{\multicolumn{3}{|c|}{$\frac{\left(5 \pm 1^{\circ} \mathrm{C}\right)}{\text { Storage durations (days) }}$}} & \multirow{3}{*}{$\begin{array}{c}5 \text { days } \\
\text { at } \\
\left(20 \pm 1^{\circ} \mathrm{C}\right)\end{array}$} & \multicolumn{3}{|c|}{$\left(7 \pm 1^{\circ} \mathrm{C}\right)$} & \multirow{3}{*}{$\begin{array}{c}5 \text { days } \\
\text { at } \\
\left(20 \pm 1^{\circ} \mathrm{C}\right)\end{array}$} \\
\hline & & & & & \multicolumn{3}{|c|}{ Storage durations (days) } & \\
\hline & 5 & 10 & 15 & & 5 & 10 & 15 & \\
\hline \multicolumn{9}{|c|}{2018 Season } \\
\hline Control & $4.00 \mathrm{~b}$ & $3.45 \mathrm{c}$ & $2.82 \mathrm{c}$ & $2.11 \mathrm{c}$ & $4.00 \mathrm{~b}$ & $3.65 c$ & -- & -- \\
\hline Lavender oil $0.25 \%$ & $5.00 \mathrm{a}$ & $4.20 \mathrm{~b}$ & $3.31 \mathrm{c}$ & $2.29 \mathrm{c}$ & $5.00 \mathrm{a}$ & $3.22 \mathrm{~b}$ & $2.45 d$ & $1.74 \mathrm{~d}$ \\
\hline Lavender oil $0.50 \%$ & $5.00 \mathrm{a}$ & $5.00 \mathrm{a}$ & $4.72 \mathrm{a}$ & $4.17 \mathrm{a}$ & $5.00 \mathrm{a}$ & $5.00 \mathrm{a}$ & $4.59 \mathrm{a}$ & $4.00 \mathrm{a}$ \\
\hline Lemongrass oil $0.25 \%$ & $5.00 \mathrm{a}$ & $4.53 a b$ & $3.86 \mathrm{bc}$ & $3.07 \mathrm{~b}$ & $5.00 \mathrm{a}$ & $3.85 b$ & $3.44 c$ & $2.87 \mathrm{c}$ \\
\hline Lemongrass oil $0.50 \%$ & $5.00 \mathrm{a}$ & $5.00 \mathrm{a}$ & $4.46 \mathrm{a}$ & $4.06 \mathrm{a}$ & $5.00 \mathrm{a}$ & $5.00 \mathrm{a}$ & $4.44 a b$ & $4.00 \mathrm{a}$ \\
\hline Thyme oil $0.25 \%$ & $4.50 a b$ & $4.11 \mathrm{bc}$ & $3.05 c$ & $1.78 \mathrm{~d}$ & $4.30 \mathrm{~b}$ & $2.74 \mathrm{c}$ & $1.98 d$ & -- \\
\hline Thyme oil $0.50 \%$ & $5.00 \mathrm{a}$ & $5.00 \mathrm{a}$ & $4.31 \mathrm{ab}$ & $3.88 \mathrm{a}$ & $5.00 \mathrm{a}$ & $4.78 \mathrm{a}$ & $4.07 \mathrm{~b}$ & $3.36 \mathrm{~b}$ \\
\hline \multicolumn{9}{|c|}{2019 Season } \\
\hline Control & $4.24 \mathrm{~b}$ & $3.22 \mathrm{~d}$ & $2.57 \mathrm{c}$ & $1.76 \mathrm{e}$ & $4.72 a b$ & $3.36 \mathrm{~d}$ & $2.27 \mathrm{~d}$ & -- \\
\hline Lavender oil $0.25 \%$ & $5.00 \mathrm{a}$ & $4.11 \mathrm{bc}$ & $3.52 b$ & $2.78 \mathrm{~d}$ & $4.67 \mathrm{~b}$ & $3.79 \mathrm{~cd}$ & $2.57 \mathrm{~cd}$ & $1.87 \mathrm{~d}$ \\
\hline Lavender oil $0.50 \%$ & $5.00 \mathrm{a}$ & $5.00 \mathrm{a}$ & $4.46 \mathrm{a}$ & $4.12 \mathrm{a}$ & $5.00 \mathrm{a}$ & $5.00 \mathrm{a}$ & $4.65 \mathrm{a}$ & $4.11 \mathrm{a}$ \\
\hline Lemongrass oil $0.25 \%$ & $5.00 \mathrm{a}$ & $4.34 \mathrm{~b}$ & $3.86 \mathrm{~b}$ & $3.14 \mathrm{c}$ & $5.00 \mathrm{a}$ & $4.21 \mathrm{bc}$ & $3.05 c$ & $2.34 \mathrm{c}$ \\
\hline Lemongrass oil $0.50 \%$ & $5.00 \mathrm{a}$ & $5.00 \mathrm{a}$ & $4.63 \mathrm{a}$ & $4.31 \mathrm{a}$ & $5.00 \mathrm{a}$ & $5.00 \mathrm{a}$ & $4.33 a b$ & $3.87 \mathrm{a}$ \\
\hline Thyme oil $0.25 \%$ & $5.00 \mathrm{a}$ & $3.54 \mathrm{~cd}$ & $2.76 \mathrm{c}$ & $1.91 \mathrm{e}$ & $4.53 \mathrm{~b}$ & $3.48 \mathrm{~d}$ & $2.00 \mathrm{~d}$ & -- \\
\hline Thyme oil $0.50 \%$ & $5.00 \mathrm{a}$ & $4.78 \mathrm{ab}$ & $4.45 \mathrm{a}$ & $3.74 \mathrm{~b}$ & $5.00 \mathrm{a}$ & $4.43 \mathrm{~b}$ & $3.84 b$ & $3.16 \mathrm{~b}$ \\
\hline
\end{tabular}

Values followed by the same letter (s) are not significantly different at $5 \%$ level 
Many studies indicated the efficiency of essential oils and plant extracts of many aromatic plants on fungal and bacterial diseases and consequently increased storability of different commodities (Lalitha et al 2011 \& Lee et al 2008). In this respect, lemongrass (Cymbopogon citratus L.) oil was reported by Hyun et al 2015, who found that an evident decrease in fungal activity against several plant pathogens and a reduction in disease incidence were obtained. However, weight loss, loss of fruit succulence and showed acceptable appearance during cold storage at market shelf conditions of avocado fruits were reported by Mpho et al 2013.

\section{2- Chemical constituents}

\section{2-1 Total chlorophyll content (mg/g f.wt)}

It is clear from data in Table (4) that total chlorophyll of mentha fresh cut herb recorded higher values with aromatic oils treatments than control. The loss of this important pigment which considered one of the main quality indicators was less in fresh cut herb-treated than untreated ones. However, storage at $5^{\circ} \mathrm{C}$ was better than storage at $7^{\circ} \mathrm{C}$ where the reduction of total chlorophyll was minimized after 15 days of storage at both temperature degrees. Data showed that after 15 days of cold storage at $5^{\circ} \mathrm{C}$, the treatments of $0.50 \%$ from the three aromatic applied oils exhibited the higher values of total chlorophyll than other treatments or control. However, after 15 days of cold storage at $7^{\circ} \mathrm{C}$ the effective treatments in preservation of total chlorophyll content from losses were the treatments of lavender oil at 0.25 or $0.50 \%$, and lemongrass at $0.25 \%$. It is important depending on the obtained data, to explain that the marketing of perishable commodities such fresh cut herb must be handling at suitable conditions $\left(20^{\circ} \mathrm{C}\right)$.

Table 4. Effect of some aromatic oils treatments on total chlorophyll content ( $\mathrm{mg} / \mathrm{g} \mathrm{f.wt)} \mathrm{herb} \mathrm{of} \mathrm{mentha} \mathrm{fresh}$ cut herb stored at $5^{\circ}$ and $7^{\circ} \mathrm{C} \pm 1$ and $95 \% \mathrm{RH}$, followed by 5 days at $20 \pm 2^{\circ} \mathrm{C}$, during 2018 and 2019 seasons

\begin{tabular}{|c|c|c|c|c|c|c|c|c|}
\hline \multirow{4}{*}{ Treatments } & \multicolumn{8}{|c|}{ Storage temperatures } \\
\hline & \multicolumn{3}{|c|}{$\left(5 \pm 1^{\circ} \mathrm{C}\right)$} & \multirow{3}{*}{$\begin{array}{c}5 \text { days } \\
\text { at } \\
\left(20 \pm 1^{\circ} \mathrm{C}\right)\end{array}$} & \multicolumn{3}{|c|}{$\left(7 \pm 1^{\circ} \mathrm{C}\right)$} & \multirow{3}{*}{$\begin{array}{c}5 \text { days } \\
\text { at } \\
\left(20 \pm 1^{\circ} \mathrm{C}\right)\end{array}$} \\
\hline & \multicolumn{3}{|c|}{ Storage durations (days) } & & \multicolumn{3}{|c|}{ Storage durations (days) } & \\
\hline & 5 & 10 & 15 & & 5 & 10 & 15 & \\
\hline \multicolumn{9}{|c|}{2018 Season } \\
\hline Control & $1.24 \mathrm{c}$ & $1.15 \mathrm{de}$ & $0.98 \mathrm{~d}$ & $0.68 \mathrm{~d}$ & $1.14 \mathrm{c}$ & $0.97 \mathrm{~d}$ & -- & -- \\
\hline Lavender oil $0.25 \%$ & $1.41 \mathrm{ab}$ & $1.27 \mathrm{c}$ & $1.03 \mathrm{c}$ & $0.83 \mathrm{c}$ & $1.29 a b$ & $1.15 b$ & $0.99 \mathrm{~b}$ & $0.83 \mathrm{c}$ \\
\hline Lavender oil $0.50 \%$ & $1.42 a b$ & $1.55 \mathrm{a}$ & $1.21 \mathrm{a}$ & $1.11 \mathrm{a}$ & $1.38 \mathrm{a}$ & $1.16 a b$ & $1.10 a b$ & $0.96 \mathrm{a}$ \\
\hline Lemongrass oil $0.25 \%$ & $1.34 \mathrm{bc}$ & $1.25 \mathrm{~cd}$ & $1.07 \mathrm{bc}$ & $0.91 \mathrm{bc}$ & $1.25 \mathrm{bc}$ & $1.07 \mathrm{bc}$ & $1.07 \mathrm{ab}$ & $0.87 \mathrm{bc}$ \\
\hline Lemongrass oil $0.50 \%$ & $1.38 \mathrm{~b}$ & $1.51 \mathrm{ab}$ & $1.22 \mathrm{a}$ & $1.02 \mathrm{a}$ & $1.36 \mathrm{ab}$ & $1.19 \mathrm{a}$ & $1.17 \mathrm{a}$ & $1.01 \mathrm{a}$ \\
\hline Thyme oil $0.25 \%$ & $1.30 \mathrm{bc}$ & $1.07 \mathrm{e}$ & $0.92 \mathrm{~d}$ & $0.62 \mathrm{~d}$ & $1.25 a b$ & $0.99 \mathrm{~cd}$ & $0.88 \mathrm{c}$ & -- \\
\hline Thyme oil $0.50 \%$ & $1.51 \mathrm{a}$ & $1.44 \mathrm{~b}$ & $1.17 a b$ & $0.97 a b$ & $1.31 \mathrm{ab}$ & $1.06 \mathrm{bc}$ & $1.05 b$ & $0.93 a b$ \\
\hline \multicolumn{9}{|c|}{2019 Season } \\
\hline Control & $1.41 \mathrm{bc}$ & $1.08 \mathrm{e}$ & $0.93 \mathrm{~d}$ & $0.63 \mathrm{e}$ & $1.18 \mathrm{~cd}$ & $0.97 \mathrm{~cd}$ & $0.85 \mathrm{c}$ & \\
\hline Lavender oil $0.25 \%$ & $1.50 a b$ & $1.35 \mathrm{bc}$ & $1.11 \mathrm{c}$ & $0.82 \mathrm{~cd}$ & $1.27 \mathrm{bc}$ & $1.02 \mathrm{c}$ & $0.99 \mathrm{~b}$ & $0.84 \mathrm{c}$ \\
\hline Lavender oil $0.50 \%$ & $1.51 \mathrm{ab}$ & $1.45 b$ & $1.37 \mathrm{a}$ & $1.00 \mathrm{~b}$ & $1.40 \mathrm{a}$ & $1.25 \mathrm{ab}$ & $1.19 \mathrm{a}$ & $1.07 \mathrm{a}$ \\
\hline Lemongrass oil $0.25 \%$ & $1.48 a b$ & $1.29 \mathrm{~cd}$ & $1.19 \mathrm{bc}$ & $0.79 \mathrm{~d}$ & $1.29 \mathrm{ab}$ & $1.15 b$ & $1.12 \mathrm{a}$ & $0.91 \mathrm{bc}$ \\
\hline Lemongrass oil $0.50 \%$ & $1.56 \mathrm{a}$ & $1.63 \mathrm{a}$ & $1.46 \mathrm{a}$ & $1.17 \mathrm{a}$ & $1.42 \mathrm{a}$ & $1.29 \mathrm{a}$ & $1.24 \mathrm{a}$ & $1.11 \mathrm{a}$ \\
\hline Thyme oil $0.25 \%$ & $1.32 \mathrm{c}$ & $1.19 \mathrm{~d}$ & $0.97 d$ & $0.75 \mathrm{de}$ & $1.11 \mathrm{~d}$ & $0.91 \mathrm{~d}$ & $0.78 \mathrm{c}$ & \\
\hline Thyme oil $0.50 \%$ & $1.49 a b$ & $1.41 \mathrm{~b}$ & $1.22 b$ & $0.90 \mathrm{c}$ & $1.34 a b$ & $1.17 b$ & $1.16 \mathrm{a}$ & $0.95 \mathrm{~b}$ \\
\hline
\end{tabular}

Initial sample for total Chlorophyll recorded 1.48 and 1.65 (mg/100 g f.wt) for 2018 and 2019 seasons

Values followed by the same letter (s) are not significantly different at $5 \%$ level 


\section{2-2 Total acidity (g citric acid / $100 \mathrm{~g}$ f.wt)}

Fresh cut herb of mentha was loss its total acidity with advanced in cold storage durations either at $5^{\circ} \mathrm{C}$ or $7^{\circ} \mathrm{C}$ (Table 5). However, after 15 days of cold storage at $5^{\circ} \mathrm{C}$, the maximum values of total acidity $(1.03 \%)$ was recorded by thyme oil at $0.25 \%$ whereas during storage at $7^{\circ} \mathrm{C}$, the highest value of total acidity (1.16\%) was exhibited by $0.25 \%$ of lavender oil treatment. Generally, it could be concluded that using aromatic oil as a pre-cold storage treatments to fresh cut herb of mentha could extend the marketing duration of the herb without high losses of total acidity. It is well known that, the reduction of total acidity with advanced in cold storage durations could be attributed to the consumption of these acids in respiration process pathway.

\section{2-3 L-ascorbic acid (mg/ g f.wt.)}

It is clear from data in Table (6) that L-ascorbic acid content in mentha fresh cut herb stored at 5 or $7^{\circ} \mathrm{C}$ for 15 days followed by 5 days at $20^{\circ} \mathrm{C}$ as a imitation marketing conditions was greatly affected by the different applied treatments. Generally, mentha fresh cut herb contained about $15 \mathrm{mg} / 100 \mathrm{~g}$ f.wt. Of $\mathrm{L}$-ascorbic acid which considered as a medium source of $L$-ascorbic acid. However, after 15 days of storage at $5^{\circ} \mathrm{C}$ the highest values of $\mathrm{L}$-ascorbic acid were recorded by $0.50 \%$ treatments of lavender, lemongrass and thyme aromatic oils treatments, and this beneficial effect was continues during marketing period at $20^{\circ} \mathrm{C}$. Regarding storage at $7^{\circ} \mathrm{C}$, lavender and thyme oils both at $0.50 \%$ exhibited higher values of $L$-ascorbic acid after 15 days of cold storage than others.

It is difficult to associate the anti-fungal activity to aromatic oils to single components or groups of compounds, generally it could explain the antifungal and antimicrobial effects to the synergistic effect (Bagamboula et al 2004).

\section{2-4 Electrolyte leakage (EC\%)}

The effect of aromatic oils treatments and cold storage temperatures on electrolyte leakage of mentha fresh cut herb are shown in Table (7). It is well known that, electrolyte leakage is considered one of good quality indicators in leafy commodities and as it increased the quality of herb is minimized. So, as cold storage durations increased either at 5 or $7^{\circ} \mathrm{C}$, electrolyte leakage values were increased regardless of the used treatments.

Table 5. Effect of some aromatic oils treatments on total acidity (g citric acid / $100 \mathrm{~g}$ f.wt.) herb \% of mentha fresh cut herb stored at $5^{\circ}$ and $7{ }^{\circ} \mathrm{C} \pm 1$ and $95 \% R H$, followed by 5 days at $20 \pm 2^{\circ} \mathrm{C}$, during 2018 and 2019 seasons

\begin{tabular}{|c|c|c|c|c|c|c|c|c|}
\hline \multirow{4}{*}{ treatments } & \multicolumn{7}{|c|}{ Storage temperatures } & \multirow{4}{*}{$\begin{array}{c}5 \text { days } \\
\text { at } \\
\left(20 \pm 1^{\circ} \mathrm{C}\right)\end{array}$} \\
\hline & \multirow{2}{*}{\multicolumn{3}{|c|}{$\begin{array}{c}\left(5 \pm 1^{\circ} \mathrm{C}\right) \\
\text { Storage durations (days) }\end{array}$}} & \multirow{3}{*}{$\begin{array}{c}5 \text { days } \\
\text { at } \\
\left(20 \pm 1^{\circ} \mathrm{C}\right)\end{array}$} & \multicolumn{3}{|c|}{$\left(7 \pm 1^{\circ} \mathrm{C}\right)$} & \\
\hline & & & & & \multicolumn{3}{|c|}{ Storage durations (days) } & \\
\hline & 5 & 10 & 15 & & 5 & 10 & 15 & \\
\hline \multicolumn{9}{|c|}{2018 Season } \\
\hline Control & $1.24 \mathrm{c}$ & $1.15 \mathrm{de}$ & $0.98 d$ & $0.68 \mathrm{~d}$ & $1.14 \mathrm{c}$ & $0.97 d$ & -- & -- \\
\hline Lavender oil $0.25 \%$ & $1.41 \mathrm{ab}$ & $1.27 \mathrm{c}$ & $1.03 \mathrm{c}$ & $0.83 \mathrm{c}$ & $1.29 \mathrm{ab}$ & $1.15 b$ & $0.99 \mathrm{~b}$ & $0.83 \mathrm{c}$ \\
\hline Lavender oil $0.50 \%$ & $1.42 a b$ & $1.55 \mathrm{a}$ & $1.21 \mathrm{a}$ & $1.11 \mathrm{a}$ & $1.38 \mathrm{a}$ & $1.16 a b$ & $1.10 a b$ & $0.96 \mathrm{a}$ \\
\hline Lemongrass oil $0.25 \%$ & $1.34 \mathrm{bc}$ & $1.25 \mathrm{~cd}$ & $1.07 \mathrm{bc}$ & $0.91 \mathrm{bc}$ & $1.25 \mathrm{bc}$ & $1.07 \mathrm{bc}$ & $1.07 \mathrm{ab}$ & $0.87 \mathrm{bc}$ \\
\hline Lemongrass oil $0.50 \%$ & $1.38 \mathrm{~b}$ & $1.51 \mathrm{ab}$ & $1.22 \mathrm{a}$ & $1.02 \mathrm{a}$ & $1.36 \mathrm{ab}$ & $1.19 \mathrm{a}$ & $1.17 \mathrm{a}$ & $1.01 \mathrm{a}$ \\
\hline Thyme oil $0.25 \%$ & $1.30 \mathrm{bc}$ & $1.07 \mathrm{e}$ & $0.92 \mathrm{~d}$ & $0.62 \mathrm{~d}$ & $1.25 a b$ & $0.99 \mathrm{~cd}$ & $0.88 \mathrm{c}$ & -- \\
\hline Thyme oil $0.50 \%$ & $1.51 \mathrm{a}$ & $1.44 \mathrm{~b}$ & $1.17 a b$ & $0.97 a b$ & $1.31 \mathrm{ab}$ & $1.06 \mathrm{bc}$ & $1.05 b$ & $0.93 \mathrm{ab}$ \\
\hline \multicolumn{9}{|c|}{2019 Season } \\
\hline Control & $1.41 \mathrm{bc}$ & $1.08 \mathrm{e}$ & $0.93 d$ & $0.63 \mathrm{e}$ & $1.18 \mathrm{~cd}$ & $0.97 \mathrm{~cd}$ & $0.85 \mathrm{c}$ & \\
\hline Lavender oil $0.25 \%$ & $1.50 a b$ & $1.35 \mathrm{bc}$ & $1.11 \mathrm{c}$ & $0.82 \mathrm{~cd}$ & $1.27 \mathrm{bc}$ & $1.02 \mathrm{c}$ & $0.99 \mathrm{~b}$ & $0.84 \mathrm{c}$ \\
\hline Lavender oil $0.50 \%$ & $1.51 \mathrm{ab}$ & $1.45 b$ & $1.37 \mathrm{a}$ & $1.00 \mathrm{~b}$ & $1.40 \mathrm{a}$ & $1.25 a b$ & $1.19 \mathrm{a}$ & $1.07 \mathrm{a}$ \\
\hline Lemongrass oil $0.25 \%$ & $1.48 a b$ & $1.29 \mathrm{~cd}$ & $1.19 \mathrm{bc}$ & $0.79 \mathrm{~d}$ & $1.29 a b$ & $1.15 b$ & $1.12 \mathrm{a}$ & $0.91 \mathrm{bc}$ \\
\hline Lemongrass oil $0.50 \%$ & $1.56 \mathrm{a}$ & $1.63 \mathrm{a}$ & $1.46 \mathrm{a}$ & $1.17 \mathrm{a}$ & $1.42 \mathrm{a}$ & $1.29 \mathrm{a}$ & $1.24 \mathrm{a}$ & $1.11 \mathrm{a}$ \\
\hline Thyme oil $0.25 \%$ & $1.32 \mathrm{c}$ & $1.19 \mathrm{~d}$ & $0.97 d$ & $0.75 \mathrm{de}$ & $1.11 \mathrm{~d}$ & $0.91 \mathrm{~d}$ & $0.78 \mathrm{c}$ & \\
\hline Thyme oil $0.50 \%$ & $1.49 a b$ & $1.41 \mathrm{~b}$ & $1.22 \mathrm{~b}$ & $0.90 \mathrm{c}$ & $1.34 a b$ & $1.17 \mathrm{~b}$ & $1.16 \mathrm{a}$ & $0.95 b$ \\
\hline
\end{tabular}

Initial sample for total acidity recorded 1.51 and 1.64 (mg/100 g f.wt) for 2018 and 2019 seasons

Values followed by the same letter (s) are not significantly different at $5 \%$ level 

of peppermint fresh cut Herb under cold storage

Table 6. Effect of some aromatic oils treatments on L- ascorbic acid ( $\mathrm{mg} / \mathrm{g}$ f.wt.) herb of mentha fresh cut herb stored at $5^{\circ}$ and $7^{\circ} \mathrm{C} \pm 1$ and $95 \% \mathrm{RH}$, followed by 5 days at $20 \pm 2^{\circ} \mathrm{C}$, during 2018 and 2019 seasons

\begin{tabular}{|c|c|c|c|c|c|c|c|c|}
\hline \multirow{4}{*}{ Treatments } & \multicolumn{8}{|c|}{ Storage temperatures } \\
\hline & \multicolumn{3}{|c|}{$\left(5 \pm 1^{\circ} \mathrm{C}\right)$} & \multirow{3}{*}{$\begin{array}{c}5 \text { days } \\
\text { at } \\
\left(20 \pm 1^{\circ} \mathrm{C}\right)\end{array}$} & \multirow{2}{*}{\multicolumn{3}{|c|}{$\frac{\left(7 \pm 1^{\circ} \mathrm{C}\right)}{\text { Storage durations (days) }}$}} & \multirow{3}{*}{$\begin{array}{c}5 \text { days } \\
\text { at } \\
\left(20 \pm 1^{\circ} \mathrm{C}\right)\end{array}$} \\
\hline & \multicolumn{3}{|c|}{ Storage durations (days) } & & & & & \\
\hline & 5 & 10 & 15 & & 5 & 10 & 15 & \\
\hline \multicolumn{9}{|c|}{2018 season } \\
\hline Control & $14.71 \mathrm{~cd}$ & $11.25 \mathrm{~d}$ & $8.75 \mathrm{e}$ & $6.23 \mathrm{~d}$ & $12.80 \mathrm{a}$ & $7.25 \mathrm{~d}$ & & \\
\hline Lavender oil $0.25 \%$ & $14.85 \mathrm{bc}$ & $14.27 \mathrm{c}$ & $12.03 \mathrm{c}$ & $9.71 \mathrm{~b}$ & $11.00 \mathrm{ab}$ & $9.75 a b$ & $7.52 \mathrm{~b}$ & $5.12 \mathrm{c}$ \\
\hline Lavender oil $0.50 \%$ & $16.43 \mathrm{a}$ & $15.72 \mathrm{a}$ & $15.11 \mathrm{a}$ & $12.22 \mathrm{a}$ & $11.51 \mathrm{ab}$ & $9.03 \mathrm{bc}$ & $7.57 \mathrm{ab}$ & $7.18 \mathrm{a}$ \\
\hline Lemongrass oil $0.25 \%$ & $14.11 \mathrm{~cd}$ & $11.55 \mathrm{~d}$ & $10.30 \mathrm{~d}$ & $7.74 \mathrm{c}$ & $10.62 \mathrm{~b}$ & $9.73 a b$ & $6.20 \mathrm{c}$ & $4.54 \mathrm{c}$ \\
\hline Lemongrass oil $0.50 \%$ & $15.74 a b$ & $13.20 \mathrm{c}$ & $13.72 b$ & $11.83 \mathrm{a}$ & $10.975 b$ & $8.73 \mathrm{bc}$ & $6.25 c$ & $7.54 \mathrm{a}$ \\
\hline Thyme oil $0.25 \%$ & $13.82 \mathrm{~d}$ & $12.27 \mathrm{~cd}$ & $9.36 \mathrm{de}$ & $7.30 \mathrm{c}$ & $12.05 \mathrm{ab}$ & $8.07 \mathrm{~cd}$ & $5.25 \mathrm{c}$ & \\
\hline Thyme oil $0.50 \%$ & $15.01 \mathrm{bc}$ & $14.44 \mathrm{~b}$ & $13.55 b$ & $10.10 \mathrm{~b}$ & $11.25 \mathrm{ab}$ & $10.05 \mathrm{a}$ & $8.53 \mathrm{a}$ & $6.11 \mathrm{~b}$ \\
\hline \multicolumn{9}{|c|}{2019 Season } \\
\hline Control & $13.31 d$ & $11.10 \mathrm{~d}$ & $7.83 d$ & $6.67 \mathrm{e}$ & $10.16 \mathrm{c}$ & $8.34 \mathrm{~d}$ & $6.11 \mathrm{c}$ & \\
\hline Lavender oil $0.25 \%$ & $14.18 \mathrm{~cd}$ & $12.22 \mathrm{c}$ & $10.41 \mathrm{c}$ & $9.63 \mathrm{c}$ & $10.12 \mathrm{c}$ & $9.26 \mathrm{~cd}$ & $8.56 \mathrm{~b}$ & $6.73 c$ \\
\hline Lavender oil $0.50 \%$ & $16.11 \mathrm{a}$ & $14.01 \mathrm{a}$ & $13.74 \mathrm{a}$ & $12.15 \mathrm{a}$ & $12.46 \mathrm{a}$ & $11.20 \mathrm{a}$ & $10.43 \mathrm{a}$ & $9.07 \mathrm{a}$ \\
\hline Lemongrass oil $0.25 \%$ & $13.58 \mathrm{~d}$ & $12.43 \mathrm{bc}$ & $10.54 \mathrm{c}$ & $7.73 \mathrm{~d}$ & $10.74 \mathrm{c}$ & $10.54 a b$ & $7.96 \mathrm{~b}$ & $5.42 \mathrm{c}$ \\
\hline Lemongrass oil $0.50 \%$ & $15.32 a b$ & $13.35 \mathrm{ab}$ & $12.20 \mathrm{~b}$ & $10.77 b$ & $12.01 \mathrm{ab}$ & $10.74 a b$ & $9.95 \mathrm{a}$ & $8.13 a b$ \\
\hline Thyme oil $0.25 \%$ & $15.03 \mathrm{~cd}$ & $12.10 \mathrm{c}$ & $8.24 d$ & $7.10 \mathrm{de}$ & $9.07 \mathrm{~d}$ & $7.54 \mathrm{~d}$ & $5.32 \mathrm{c}$ & \\
\hline Thyme oil $0.50 \%$ & $14.91 \mathrm{bc}$ & $12.92 \mathrm{bc}$ & $11.14 \mathrm{c}$ & $9.47 \mathrm{c}$ & $11.09 \mathrm{bc}$ & $9.75 \mathrm{bc}$ & $8.24 \mathrm{~b}$ & $7.93 \mathrm{~b}$ \\
\hline
\end{tabular}

Initial sample for recorded 16.15 and 16.83 (mg/ $100 \mathrm{~g}$ f.wt.) for 2018 and 2019 seasons

Values followed by the same letter (s) are not significantly different at $5 \%$ level

Table 7. Effect of some aromatic oils treatments on electrolyte leakage (EC\%) herb of mentha fresh cut herb stored at $5^{\circ}$ and $7{ }^{\circ} \mathrm{C} \pm 1$ and $95 \% \mathrm{RH}$, followed by 5 days at $20 \pm 2^{\circ} \mathrm{C}$, during 2018 and 2019 seasons

\begin{tabular}{|c|c|c|c|c|c|c|c|c|}
\hline \multirow{4}{*}{ Treatments } & \multicolumn{8}{|c|}{ Storage temperatures } \\
\hline & \multicolumn{3}{|c|}{$\left(5 \pm 1^{\circ} \mathrm{C}\right)$} & \multirow{3}{*}{$\begin{array}{c}5 \text { days } \\
\text { at } \\
\left(20 \pm 1^{\circ} \mathrm{C}\right)\end{array}$} & \multicolumn{3}{|c|}{$\left(7 \pm 1^{\circ} \mathrm{C}\right)$} & \multirow{3}{*}{$\begin{array}{c}5 \text { days } \\
\text { at } \\
\left(20 \pm 1^{\circ} \mathrm{C}\right)\end{array}$} \\
\hline & \multicolumn{3}{|c|}{ Storage durations (days) } & & \multicolumn{3}{|c|}{ Storage durations (days) } & \\
\hline & 5 & 10 & 15 & & 5 & 10 & 15 & \\
\hline \multicolumn{9}{|c|}{2018 season } \\
\hline Control & $22.56 \mathrm{c}$ & $29.15 b c$ & $43.57 \mathrm{a}$ & $71.11 \mathrm{a}$ & $28.75 a$ & $42.29 a$ & --- & -- \\
\hline Lavender oil $0.25 \%$ & $20.27 d$ & $26.26 \mathrm{e}$ & $32.17 \mathrm{~d}$ & $37.13 \mathrm{e}$ & $26.20 \mathrm{~b}$ & $28.74 \mathrm{e}$ & $35.20 \mathrm{~d}$ & $61.12 \mathrm{~d}$ \\
\hline Lavender oil $0.50 \%$ & $25.74 \mathrm{a}$ & $28.95 \mathrm{~cd}$ & 39.74 b & $56.11 \mathrm{~d}$ & 29.42 a & $35.11 \mathrm{c}$ & $42.79 \mathrm{~b}$ & $71.11 \mathrm{a}$ \\
\hline Lemongrass oil $0.25 \%$ & $20.16 d$ & $28.02 \mathrm{~d}$ & $35.54 \mathrm{c}$ & $40.27 \mathrm{e}$ & 24.27 c & $29.19 \mathrm{e}$ & $40.27 \mathrm{bc}$ & $61.23 \mathrm{~cd}$ \\
\hline Lemongrass oil $0.50 \%$ & $21.15 \mathrm{~d}$ & $27.23 \mathrm{e}$ & $36.11 \mathrm{c}$ & $60.20 \mathrm{c}$ & $26.44 \mathrm{~b}$ & $32.29 d$ & $41.19 \mathrm{~b}$ & $66.53 \mathrm{~b}$ \\
\hline Thyme oil $0.25 \%$ & $24.11 b$ & $32.59 \mathrm{a}$ & $46.25 \mathrm{a}$ & $67.33 \mathrm{~b}$ & $28.71 \mathrm{a}$ & $38.56 \mathrm{~b}$ & $54.20 \mathrm{a}$ & -- \\
\hline Thyme oil $0.50 \%$ & $21.92 \mathrm{~cd}$ & $30.00 \mathrm{~b}$ & $38.32 \mathrm{bc}$ & $53.14 \mathrm{~d}$ & $28.50 \mathrm{a}$ & $31.37 d$ & $38.12 \mathrm{c}$ & $64.11 \mathrm{bc}$ \\
\hline \multicolumn{9}{|c|}{2019 Season } \\
\hline Control & $23.19 \mathrm{a}$ & $34.41 \mathrm{a}$ & $49.30 \mathrm{a}$ & $70.16 \mathrm{a}$ & $24.71 \mathrm{a}$ & $33.75 \mathrm{a}$ & $49.66 \mathrm{a}$ & \\
\hline Lavender oil $0.25 \%$ & $23.31 \mathrm{a}$ & $29.30 \mathrm{~b}$ & $41.44 \mathrm{bc}$ & $60.21 \mathrm{~cd}$ & $24.41 \mathrm{a}$ & $30.07 \mathrm{~b}$ & $43.18 \mathrm{~cd}$ & 70.40 a \\
\hline Lavender oil $0.50 \%$ & $19.10 \mathrm{c}$ & $21.91 \mathrm{~d}$ & $33.30 \mathrm{de}$ & $46.01 \mathrm{e}$ & $21.34 d$ & $24.66 \mathrm{~d}$ & $38.78 \mathrm{e}$ & $53.76 \mathrm{e}$ \\
\hline Lemongrass oil $0.25 \%$ & $21.43 b$ & $29.47 b$ & $39.61 \mathrm{c}$ & $63.33 \mathrm{c}$ & $23.22 \mathrm{bc}$ & $29.28 b$ & $43.63 \mathrm{bc}$ & $68.32 \mathrm{~b}$ \\
\hline Lemongrass oil $0.50 \%$ & $17.08 \mathrm{~d}$ & $22.63 d$ & $30.63 \mathrm{e}$ & $48.05 \mathrm{e}$ & $21.56 \mathrm{~d}$ & $25.19 d$ & $37.36 \mathrm{e}$ & $56.10 \mathrm{~d}$ \\
\hline Thyme oil $0.25 \%$ & $23.76 \mathrm{a}$ & 29.92 b & $43.38 \mathrm{~b}$ & $64.79 \mathrm{~b}$ & $24.06 \mathrm{ab}$ & $32.58 \mathrm{a}$ & $46.46 \mathrm{~b}$ & \\
\hline Thyme oil $0.50 \%$ & $20.39 c$ & $24.73 \mathrm{c}$ & $35.81 \mathrm{~d}$ & $56.87 \mathrm{~d}$ & $22.52 \mathrm{~cd}$ & $26.38 \mathrm{c}$ & 39.74 de & $62.15 \mathrm{c}$ \\
\hline
\end{tabular}

Initial sample for Electrolyte Leakage recorded 18.79 and 16.11 (EC\%) for 2018 and 2019 seasons

Values followed by the same letter (s) are not significantly different at $5 \%$ level 
However, after 15 days of cold storage at $5^{\circ} \mathrm{C}$ the least value of electrolyte leakage $(32.17 \%)$ was recorded by lavender oil treatment at $0.25 \%$ and this effect was also found after 5 days at $20^{\circ} \mathrm{C}$ as a simulation to ideal marketing conditions. On the other hand, after 15 days of storage at $7^{\circ} \mathrm{C}$ the same treatment of lavender at $0.25 \%$ recorded the least value of electrolyte leakage (35.20\%) and the reducing effect was continuous at $20^{\circ} \mathrm{C}$ for 5 days. Generally, electrolyte leakage values were higher during storage at $7^{\circ} \mathrm{C}$ than $5^{\circ} \mathrm{C}$ which means that $5^{\circ} \mathrm{C}$ storage temperature was suitable for menthe fresh cut herb than $7^{\circ} \mathrm{C}$.

Due to large distances that the product generally must transport between the location of production and the shelf-life of the consumer, the demand for postharvest techniques to maintain the quality of the fresh product for longer of time has grown (Wills et al 2007).

\section{2-5 Total phenols ( $\mathrm{mg} / \mathbf{1 0 0 g}$ d.wt.)}

As it shown in Table (8), it is clear that total phenols content of mentha fresh cut herb was decreased with advanced in cold storage durations either at 5 or $7^{\circ} \mathrm{C}$. The treatments of lemongrass oil at $0.25 \%$ and thyme oil at $0.50 \%$ were effective than other treatments or control in reducing the rate of loss in total phenols and recorded the highest values after 15 days of cold storage at $5^{\circ} \mathrm{C}$. However, lavender oil and lemongrass oil both at $0.50 \%$ were recorded the highest values of total phenols after 15 days of cold storage at $7^{\circ} \mathrm{C}$.

In addition, during marketing durations at $20^{\circ} \mathrm{C}$, the treatment of $0.50 \%$ lavender oil was effective in recording the highest values of total phenols after finished from cold storage process either at $5^{\circ}$ or $7^{\circ}$ storage temperatures.

Table 8. Effect of some aromatic oils treatments on total phenols ( $\mathrm{mg} / 100 \mathrm{~g} \mathrm{~d}$.wt.) herb of mentha fresh cut herb stored at $5^{\circ}$ and $7^{\circ} \mathrm{C} \pm 1$ and $95 \% \mathrm{RH}$, followed by 5 days at $20 \pm 2^{\circ} \mathrm{C}$, during 2018 and 2019 season

\begin{tabular}{|c|c|c|c|c|c|c|c|c|}
\hline \multirow{4}{*}{ Treatments } & \multicolumn{8}{|c|}{ Storage temperatures } \\
\hline & \multirow{2}{*}{\multicolumn{3}{|c|}{$\frac{\left(5 \pm 1^{\circ} \mathrm{C}\right)}{\text { Storage durations (days) }}$}} & \multirow{3}{*}{$\begin{array}{c}5 \text { days } \\
\text { at } \\
\left(20 \pm 1^{\circ} \mathrm{C}\right)\end{array}$} & \multicolumn{3}{|c|}{$\left(7 \pm 1^{\circ} \mathrm{C}\right)$} & \multirow{3}{*}{$\begin{array}{c}5 \text { days } \\
\text { at } \\
\left(20 \pm 1^{\circ} \mathrm{C}\right)\end{array}$} \\
\hline & & & & & \multicolumn{3}{|c|}{ Storage durations (days) } & \\
\hline & 5 & 10 & 15 & & 5 & 10 & 15 & \\
\hline \multicolumn{9}{|c|}{2018 season } \\
\hline Control & $35.49 \mathrm{~d}$ & $32.85 \mathrm{c}$ & $28.70 \mathrm{bc}$ & $18.00 \mathrm{~d}$ & $27.90 \mathrm{~d}$ & $25.45 \mathrm{~b}$ & -- & -- \\
\hline Lavender oil $0.25 \%$ & $40.04 \mathrm{c}$ & $32.41 \mathrm{c}$ & 28 & $22.12 \mathrm{c}$ & $28.27 \mathrm{~cd}$ & $24.39 \mathrm{c}$ & $19.67 \mathrm{~d}$ & $14.05 \mathrm{e}$ \\
\hline Lavender oil $0.50 \%$ & $39.07 \mathrm{c}$ & $31.57 \mathrm{c}$ & 29.78 bc & $28.06 \mathrm{a}$ & $28.69 \mathrm{~cd}$ & $24.17 c$ & $22.16 \mathrm{a}$ & $21.27 \mathrm{a}$ \\
\hline Lemongrass oil $0.25 \%$ & $44.25 \mathrm{a}$ & $38.62 \mathrm{a}$ & $35.72 \mathrm{a}$ & $25.86 b$ & $29.76 \mathrm{bc}$ & $25.45 b$ & $19.45 d$ & $16.17 \mathrm{~d}$ \\
\hline Lemongrass oil $0.50 \%$ & $43.38 \mathrm{~b}$ & $36.39 \mathrm{~b}$ & $30.37 \mathrm{~b}$ & $25.46 \mathrm{~b}$ & $30.44 \mathrm{ab}$ & $25.44 \mathrm{~b}$ & $22.10 \mathrm{ab}$ & $19.87 b$ \\
\hline Thyme oil $0.25 \%$ & $33.43 \mathrm{~d}$ & $28.56 \mathrm{~d}$ & $22.33 d$ & $19.93 d$ & $29.35 \mathrm{bc}$ & $25.59 \mathrm{~b}$ & $21.11 \mathrm{bc}$ & -- \\
\hline Thyme oil $0.50 \%$ & $44.96 \mathrm{ab}$ & $32.88 \mathrm{c}$ & $34.02 \mathrm{a}$ & $24.80 \mathrm{~b}$ & $31.77 \mathrm{a}$ & $28.65 \mathrm{a}$ & $20.61 \mathrm{~cd}$ & $18.18 \mathrm{c}$ \\
\hline \multicolumn{9}{|c|}{2019 Season } \\
\hline Control & $36.75 \mathrm{bc}$ & $28.41 \mathrm{~cd}$ & $21.72 \mathrm{c}$ & $16.66 \mathrm{e}$ & $29.32 \mathrm{~d}$ & $25.11 \mathrm{bc}$ & $21.57 \mathrm{a}$ & -- \\
\hline Lavender oil $0.25 \%$ & $37.15 \mathrm{bc}$ & $29.55 \mathrm{bc}$ & $22.34 \mathrm{c}$ & $20.11 d$ & $30.15 \mathrm{~cd}$ & $23.20 d$ & $16.56 \mathrm{~d}$ & $12.31 \mathrm{~d}$ \\
\hline Lavender oil $0.50 \%$ & $42.38 \mathrm{a}$ & $34.45 \mathrm{a}$ & $30.11 \mathrm{a}$ & $29.74 \mathrm{a}$ & $32.95 \mathrm{a}$ & $28.56 \mathrm{a}$ & $21.08 \mathrm{a}$ & $19.91 \mathrm{a}$ \\
\hline Lemongrass oil $0.25 \%$ & $38.25 b$ & $31.10 \mathrm{~b}$ & $24.40 \mathrm{~b}$ & $21.83 \mathrm{~cd}$ & $31.75 a b$ & $25.11 \mathrm{bc}$ & $15.59 \mathrm{~d}$ & $14.54 \mathrm{c}$ \\
\hline Lemongrass oil $0.50 \%$ & $35.56 \mathrm{c}$ & $27.21 \mathrm{de}$ & $21.47 c$ & $24.26 b$ & $30.16 \mathrm{~cd}$ & $23.74 \mathrm{~d}$ & $19.58 b$ & $18.44 \mathrm{a}$ \\
\hline Thyme oil $0.25 \%$ & $35.54 \mathrm{c}$ & $30.53 \mathrm{e}$ & $16.74 \mathrm{~d}$ & $14.71 \mathrm{e}$ & $30.85 \mathrm{bc}$ & $25.26 \mathrm{~b}$ & $18.45 \mathrm{c}$ & -- \\
\hline Thyme oil $0.50 \%$ & $36.26 \mathrm{bc}$ & $30.27 \mathrm{bc}$ & $26.70 \mathrm{~b}$ & $23.20 \mathrm{bc}$ & $31.56 \mathrm{ab}$ & $24.25 \mathrm{~cd}$ & $19.51 \mathrm{~b}$ & $16.66 \mathrm{~b}$ \\
\hline
\end{tabular}

Initial sample for total phenols recorded 45.51 and 43.92 (mg /100 g d.wt.) for 2018 and 2019 seasons Values followed by the same letter (s) are not significantly different at $5 \%$ level 
A great effect of phenolic compounds was observed by (Karthikesan et al 2010) through of hepatoprotective and hypolipidemic activities by inhibition lipid peroxidation and antioxidant properties. In addition, Brunerton 2005, reported that phenols compounds has a wide spectrum of biological activities including anti-inflamantory, anti-microbial, antioxidant and immunomodulatory properties.

\section{2-6 Total flavones (mg/100g d.wt)}

Table (9) data declared that total flavones of mentha fresh cut herb was greatly affected by both cold storage conditions and aromatic oil treatments. However, lavender, lemongrass and thyme aromatic oils treatments all at $0.50 \%$ recorded the highest values of total flavones after 15 days of cold storage either at $5^{\circ} \mathrm{C}$ or $7^{\circ} \mathrm{C}$. However, during marketing durations at $20^{\circ} \mathrm{C}$ for 5 days after finished from cold storage at $5^{\circ} \mathrm{C}$ or $7^{\circ} \mathrm{C}$, it is noticed that storage of fresh cut herb at $5^{\circ} \mathrm{C}$ was better than those stored at $7^{\circ} \mathrm{C}$, where it exhibited higher values of total flavones. It is well known that, total flavones considered as a pre cursor of many vitamins and consequently increased the nutritive value of herb. From the obtained data it could be concluded that the used of aromatic oils as a pre-storage treatments had many benefits mainly as environmental safe treatments.

The benefits of mentha different species had many health benefits, nutritional supplement in livestock feed (Hosoda et al 2005), food and mouth freshener. So, since ancient times mint species are being consumed without any side effect and they are generally considered safe for human and animal consumption due to they are rich in polyphenols and flavones in different form. These main components are important in human health as a source for vitamins and as antioxidant for radical free (Maisuthisakul et al 2007).

Table 9. Effect of some aromatic oils treatments on total flavones (mg / $100 \mathrm{~g} \mathrm{~d}$.wt.) herb of mentha fresh cut herb stored at $5^{\circ}$ and $7{ }^{\circ} \mathrm{C} \pm 1$ and $95 \% \mathrm{RH}$, followed by 5 days at $20 \pm 2{ }^{\circ} \mathrm{C}$, during 2018 and 2019 seasons

\begin{tabular}{|c|c|c|c|c|c|c|c|c|}
\hline \multirow{4}{*}{ Treatments } & \multicolumn{8}{|c|}{ Storage temperatures } \\
\hline & \multicolumn{3}{|c|}{$\left(5 \pm 1^{\circ} \mathrm{C}\right)$} & \multirow{3}{*}{$\begin{array}{c}5 \text { days } \\
\text { at } \\
\left(20 \pm 1^{\circ} \mathrm{C}\right)\end{array}$} & \multicolumn{3}{|c|}{$\left(7 \pm 1^{\circ} \mathrm{C}\right)$} & \multirow{3}{*}{$\begin{array}{c}5 \text { days } \\
\text { at } \\
\left(20 \pm 1^{\circ} \mathrm{C}\right)\end{array}$} \\
\hline & \multicolumn{3}{|c|}{ Storage durations (days) } & & \multicolumn{3}{|c|}{ Storage durations (days) } & \\
\hline & 5 & 10 & 15 & & 5 & 10 & 15 & \\
\hline \multicolumn{9}{|c|}{2018 season } \\
\hline Control & $23.11 \mathrm{e}$ & $18.23 \mathrm{~cd}$ & $11.55 \mathrm{e}$ & $7.75 \mathrm{f}$ & $20.51 \mathrm{~cd}$ & $17.21 \mathrm{~d}$ & -- & -- \\
\hline Lavender oil $0.25 \%$ & 25. $23 \mathrm{de}$ & 19.32 c & $19.17 \mathrm{c}$ & $17.17 \mathrm{~d}$ & $21.49 \mathrm{bcd}$ & $19.34 \mathrm{c}$ & $15.20 \mathrm{~b}$ & $9.75 \mathrm{c}$ \\
\hline Lavender oil $0.50 \%$ & $29.7 a b$ & $23.55 \mathrm{a}$ & $23.46 \mathrm{a}$ & $22.17 \mathrm{a}$ & $22.78 a b$ & $21.56 \mathrm{a}$ & $18.17 \mathrm{a}$ & $12.74 \mathrm{a}$ \\
\hline Lemongrass oil $0.25 \%$ & $26.30 \mathrm{~cd}$ & $18.10 \mathrm{~cd}$ & $18.56 \mathrm{c}$ & $19.19 \mathrm{c}$ & $21.93 \mathrm{bc}$ & $20.38 \mathrm{~b}$ & $14.34 \mathrm{~b}$ & $14.21 \mathrm{a}$ \\
\hline Lemongrass oil $0.50 \%$ & $30.58 \mathrm{a}$ & $21.36 \mathrm{~b}$ & $22.10 \mathrm{ab}$ & $21.25 \mathrm{a}$ & $23.90 \mathrm{a}$ & $21.74 \mathrm{a}$ & $17.20 \mathrm{a}$ & $14.35 b$ \\
\hline Thyme oil $0.25 \%$ & $20.17 f$ & $17.10 \mathrm{~d}$ & $13.23 d$ & $11.12 \mathrm{e}$ & $20.41 d$ & $18.21 d$ & $13.74 \mathrm{c}$ & -- \\
\hline Thyme oil $0.50 \%$ & $28.26 \mathrm{bc}$ & $22.71 \mathrm{ab}$ & $21.59 \mathrm{~b}$ & $20.63 \mathrm{~b}$ & $22.06 \mathrm{~b}$ & $21.56 \mathrm{a}$ & $18.13 \mathrm{a}$ & $14.51 \mathrm{a}$ \\
\hline \multicolumn{9}{|c|}{2019 Season } \\
\hline Control & $24.10 \mathrm{~d}$ & $22.57 \mathrm{bc}$ & $10.40 \mathrm{e}$ & $8.57 \mathrm{e}$ & $23.51 \mathrm{~d}$ & $17.20 \mathrm{e}$ & $11.57 \mathrm{e}$ & -- \\
\hline Lavender oil $0.25 \%$ & $24.25 \mathrm{~cd}$ & $24.59 \mathrm{a}$ & $17.20 \mathrm{c}$ & $16.17 \mathrm{c}$ & $26.11 \mathrm{c}$ & $24.20 \mathrm{c}$ & $19.17 \mathrm{c}$ & $12.25 \mathrm{~d}$ \\
\hline Lavender oil $0.50 \%$ & $28.75 \mathrm{a}$ & $25.10 \mathrm{a}$ & $22.20 \mathrm{a}$ & $20.46 \mathrm{a}$ & 30.07 a & $27.58 \mathrm{a}$ & $23.46 \mathrm{a}$ & $16.13 \mathrm{a}$ \\
\hline Lemongrass oil $0.25 \%$ & $25.07 \mathrm{~cd}$ & $20.44 d$ & $19.14 b$ & $15.56 \mathrm{c}$ & $27.56 \mathrm{bc}$ & $23.59 \mathrm{c}$ & $18.56 \mathrm{c}$ & $14.36 \mathrm{c}$ \\
\hline Lemongrass oil $0.50 \%$ & $28.11 \mathrm{ab}$ & $24.73 \mathrm{a}$ & $21.33 \mathrm{a}$ & $19.10 \mathrm{ab}$ & $28.52 a b$ & $26.43 a b$ & $22.10 a b$ & $15.28 \mathrm{ab}$ \\
\hline Thyme oil $0.25 \%$ & $24.25 \mathrm{~cd}$ & $21.57 \mathrm{~cd}$ & $14.56 \mathrm{~d}$ & $10.25 d$ & $26.73 \mathrm{c}$ & $21.51 \mathrm{~d}$ & $13.25 d$ & -- \\
\hline Thyme oil $0.50 \%$ & $26.15 \mathrm{bc}$ & $24.32 \mathrm{ab}$ & $20.11 b$ & $18.59 \mathrm{~b}$ & $27.73 \mathrm{bc}$ & $24.95 b c$ & $21.59 \mathrm{~b}$ & $15.11 \mathrm{bc}$ \\
\hline
\end{tabular}

Initial sample for total flavones recorded 32.27 and 29.81 (mg / $100 \mathrm{~g}$ d.wt.) for 2018 and 2019 seasons

Values followed by the same letter (s) are not significantly different at $5 \%$ level 


\section{Respiration rate $\left(\mathrm{mg} \mathrm{CO}_{2} / \mathrm{kg}\right.$ herb/hr.)}

Table (10) data suggested that the supplementary refrigeration treatments including 3 aromatic oils at two rates were effective in minimizing the respiration rate of menthe fresh cut herb. After 15 days of cold storage at $5^{\circ} \mathrm{C}$, the treatments of high concentrations $(0.50 \%)$ from the three aromatic oils were recorded the least values of respiration rate followed by the low one $(0.25 \%)$. Meanwhile, the same trend of the results was also obtained after 15 days of cold storage at $7^{\circ} \mathrm{C}$, but with high values of respiration rate than those on $5^{\circ} \mathrm{C}$. This finding led to more preservation quality of fresh cut herb during storage at $5^{\circ} \mathrm{C}$ than at $7^{\circ} \mathrm{C}$. During marketing period at $20^{\circ} \mathrm{C}$ for 5 days, the effect of volatile oils treatments were also reported, where all oils treatments recorded less respiration rate values than control either after cold storage at 5 or $7^{\circ} \mathrm{C}$. The great effect of low temperature on reducing the respiration rate of fresh herbs was also mentioned by (Sandhya, 2010). In similar findings, thyme (Thymus cupitates L.) oil has been used to control many plant diseases of several fruits (Abd-alla et al 2011). It was effective in preserved the losses of vitamin $C$ and maintaining quality of the orange fruits (Fatemi et al 2011). Also, bergamot (Citrus bergamia) oil minimized fruit decay, weight loss \%, delayed the change in fruit softening, total acidity, T.S.S\%, vitamin $\mathrm{C}$, anthocyamin pigment content and respiration rate of Crimson seedless grapes (Abd-Elwahab et al 2014).

Table 10. Effect of some aromatic oils treatments on respiration rate $\left(\mathrm{mg} \mathrm{CO}_{2} / \mathrm{kg}\right.$ herb / hr.) herb of mentha fresh cut herb stored at $5^{\circ}$ and $7{ }^{\circ} \mathrm{C} \pm 1$ and $95 \% \mathrm{RH}$, followed by 5 days at $20 \pm 2{ }^{\circ} \mathrm{C}$, during 2018 and 2019 seasons

\begin{tabular}{|c|c|c|c|c|c|c|c|c|}
\hline \multirow{4}{*}{ Treatments } & \multicolumn{8}{|c|}{ Storage temperatures } \\
\hline & \multicolumn{3}{|c|}{$\left(5 \pm 1^{\circ} \mathrm{C}\right)$} & \multirow{3}{*}{$\begin{array}{c}5 \text { days } \\
\text { at } \\
\left(20 \pm 1^{\circ} \mathrm{C}\right)\end{array}$} & \multicolumn{3}{|c|}{$\left(7 \pm 1^{\circ} \mathrm{C}\right)$} & \multirow{3}{*}{$\begin{array}{c}5 \text { days } \\
\text { at } \\
\left(20 \pm 1^{\circ} \mathrm{C}\right)\end{array}$} \\
\hline & \multicolumn{3}{|c|}{ Storage durations (days) } & & \multicolumn{3}{|c|}{ Storage durations (days) } & \\
\hline & 5 & 10 & 15 & & 5 & 10 & 15 & \\
\hline \multicolumn{9}{|c|}{2018 season } \\
\hline Control & $32.69 \mathrm{c}$ & $39.25 \mathrm{~d}$ & $37.82 \mathrm{e}$ & $71.87 \mathrm{e}$ & $47.73 \mathrm{a}$ & $56.73 \mathrm{~b}$ & -- & -- \\
\hline Lavender oil $0.25 \%$ & $35.21 \mathrm{bc}$ & $40.11 \mathrm{~cd}$ & $45.94 \mathrm{c}$ & $96.35 d$ & $26.18 \mathrm{~d}$ & $38.84 \mathrm{~d}$ & $58.54 \mathrm{~d}$ & $104.43 d$ \\
\hline Lavender oil $0.50 \%$ & $39.93 \mathrm{a}$ & $56.51 \mathrm{a}$ & $54.94 b$ & $133.40 \mathrm{a}$ & $41.83 \mathrm{~b}$ & $55.12 \mathrm{~b}$ & $72.00 \mathrm{~b}$ & $145.30 \mathrm{a}$ \\
\hline Lemongrass oil $0.25 \%$ & $38.73 \mathrm{a}$ & $49.53 \mathrm{~b}$ & $53.76 \mathrm{~b}$ & $98.80 \mathrm{~cd}$ & $26.50 \mathrm{~d}$ & $43.46 \mathrm{c}$ & 53.82 e & $115.19 \mathrm{c}$ \\
\hline Lemongrass oil $0.50 \%$ & $38.11 \mathrm{ab}$ & $54.30 \mathrm{a}$ & $63.48 \mathrm{a}$ & $112.71 \mathrm{~b}$ & $38.15 \mathrm{~b}$ & $56.70 \mathrm{~b}$ & $72.03 \mathrm{~b}$ & $139.35 \mathrm{a}$ \\
\hline Thyme oil $0.25 \%$ & $33.24 \mathrm{c}$ & $40.25 \mathrm{~cd}$ & $41.08 \mathrm{~d}$ & $75.61 \mathrm{e}$ & $46.26 \mathrm{a}$ & $60.78 \mathrm{a}$ & $80.22 \mathrm{a}$ & -- \\
\hline Thyme oil $0.50 \%$ & $36.65 \mathrm{ab}$ & $42.56 \mathrm{c}$ & $54.08 \mathrm{~b}$ & $104.73 \mathrm{c}$ & $31.20 \mathrm{c}$ & $46.35 \mathrm{c}$ & $64.25 \mathrm{c}$ & $128.60 \mathrm{~b}$ \\
\hline \multicolumn{9}{|c|}{2019 Season } \\
\hline Control & $30.56 \mathrm{~b}$ & $41.73 \mathrm{~d}$ & $57.57 \mathrm{~d}$ & $84.53 \mathrm{e}$ & $46.66 \mathrm{a}$ & $66.54 \mathrm{~b}$ & $76.60 \mathrm{a}$ & -- \\
\hline Lavender oil $0.25 \%$ & $35.35 \mathrm{a}$ & $46.25 \mathrm{c}$ & $69.55 \mathrm{~b}$ & $105.94 \mathrm{c}$ & $40.00 \mathrm{~b}$ & $48.17 \mathrm{de}$ & $49.44 \mathrm{e}$ & $120.81 \mathrm{~d}$ \\
\hline Lavender oil $0.50 \%$ & $37.22 \mathrm{a}$ & $59.20 \mathrm{a}$ & $78.72 \mathrm{a}$ & $135.19 \mathrm{a}$ & $46.10 \mathrm{a}$ & $57.22 \mathrm{c}$ & $70.07 \mathrm{~b}$ & $156.81 \mathrm{a}$ \\
\hline Lemongrass oil $0.25 \%$ & $36.30 \mathrm{a}$ & $51.43 b$ & $70.18 b$ & $111.00 \mathrm{bc}$ & $40.23 \mathrm{~b}$ & $46.71 \mathrm{e}$ & $46.01 \mathrm{e}$ & $113.78 \mathrm{~d}$ \\
\hline Lemongrass oil $0.50 \%$ & $34.98 \mathrm{a}$ & $61.10 \mathrm{a}$ & $76.71 \mathrm{a}$ & $131.57 \mathrm{a}$ & $45.11 \mathrm{a}$ & $51.19 \mathrm{~d}$ & $60.56 \mathrm{~d}$ & $148.56 \mathrm{~b}$ \\
\hline Thyme oil $0.25 \%$ & $31.27 b$ & $38.00 \mathrm{e}$ & $52.48 \mathrm{e}$ & $92.12 \mathrm{~d}$ & $44.62 \mathrm{a}$ & $71.64 \mathrm{a}$ & 64.86 c & \\
\hline Thyme oil $0.50 \%$ & $31.95 \mathrm{~b}$ & $52.50 \mathrm{~b}$ & $62.34 \mathrm{c}$ & $115.28 b$ & $41.57 \mathrm{~b}$ & $49.96 \mathrm{~d}$ & $66.64 \mathrm{bc}$ & $135.60 \mathrm{c}$ \\
\hline
\end{tabular}

Initial sample for total flavones recorded 7288 and $78.19\left(\mathrm{mg} \mathrm{CO}_{2} / \mathrm{kg} / \mathrm{hr}\right.$.) for 2018 and 2019 seasons

Values followed by the same letter (s) are not significantly different at $5 \%$ level

\section{Volatile oil \%}

A great effect of both supplementary refrigeration treatments in the present study, i.e. volatile oils treatments and cold storage conditions on volatile oil \% of mentha fresh cut herb was illustrated in Table (11).

Hence, volatile oil \% in mentha fresh cut herb ranged from $0.26-0.27 \%$ F.wt. regardless of the used treatments or storage conditions. However, after 15 days of cold storage at $5^{\circ} \mathrm{C}$ or $7^{\circ} \mathrm{C}$, the highest value of volatile oil \% $(0.20 \%)$ were recorded by $0.50 \%$ of lavender oil treatment . In addition, during marketing durations for 5 days at $20^{\circ} \mathrm{C}$ after finished from cold storage process at $5^{\circ}$ or $7^{\circ} \mathrm{C}$, the great affect to $0.50 \%$ lavender oil treatment was also obtained. Volatile oil of menthe has used in many purpose like perfume industry, insecticidal treatments 
and as a cumulative (Ghorbani and Esmaeilizadeh, 2017). Finally, it could be concluded that fresh cut herb and perishable commodities must handle and marketing under cold conditions $\left(20^{\circ} \mathrm{C}\right)$ to reduce the loss of active ingredient and increase of shelf-life.

In this regard, Wang 2003 reported that treating raspberries with natural volatile compounds increased fruit acidity during cold storage. However, application of lemongrass aromatic oil significantly preserved potato quality i.e. minimize sprouting percentage, sprout number, length of sprout and weight loss during cold storage (Kushal et al 2020). Similar trends of results on the great effect of aromatic oils on postharvest quality as a supplementary refrigeration treatments were also found with peppermint oil on orange fruits (Fatemi et al 2011) and plum fruits (Aminifard and Mohammadi, 2013), rosemary oil on many commodities (Almela et al 2006), oils of lavender, dill and coriander (Serban et al 2011). The role of essential oils as antibiotic activity, allelopathy, attractants, feed in deterrents and phytoalexin was reported by Mahanta et al 2007.

Table 11. Effect of some aromatic oils treatments on volatile oil herb \% of mentha fresh cut herb stored at $5^{\circ}$ and $7{ }^{\circ} \mathrm{C} \pm 1$ and $95 \% \mathrm{RH}$, followed by 5 days at $20 \pm 2^{\circ} \mathrm{C}$, during 2018 and 2019 seasons

\begin{tabular}{|c|c|c|c|c|c|c|c|c|}
\hline \multirow{4}{*}{ Treatments } & & & & \multicolumn{5}{|c|}{ Storage temperatures } \\
\hline & \multicolumn{3}{|c|}{\begin{tabular}{|c|}
$\left(5 \pm 1^{\circ} \mathrm{C}\right)$ \\
\end{tabular}} & \multirow{3}{*}{$\begin{array}{c}5 \text { days } \\
\text { at } \\
\left(20 \pm 1^{\circ} \mathrm{C}\right)\end{array}$} & \multicolumn{3}{|c|}{$\left(7 \pm 1^{\circ} \mathrm{C}\right)$} & \multirow{3}{*}{$\begin{array}{c}5 \text { days } \\
\text { at } \\
\left(20 \pm 1^{\circ} \mathrm{C}\right)\end{array}$} \\
\hline & \multicolumn{3}{|c|}{ Storage durations (days) } & & \multicolumn{3}{|c|}{ Storage durations (days) } & \\
\hline & 5 & 10 & 15 & & 5 & 10 & 15 & \\
\hline \multicolumn{9}{|c|}{2018 season } \\
\hline Control & $0.20 \mathrm{~d}$ & $0.19 \mathrm{c}$ & $0.12 \mathrm{~d}$ & $0.10 \mathrm{~d}$ & $0.19 \mathrm{~d}$ & $0.16 \mathrm{c}$ & -- & -- \\
\hline Lavender oil $0.25 \%$ & $0.24 \mathrm{c}$ & $0.20 \mathrm{bc}$ & $0.17 \mathrm{bc}$ & $0.12 \mathrm{~cd}$ & $0.25 a b$ & $0.23 \mathrm{a}$ & $0.20 \mathrm{a}$ & $0.10 \mathrm{~d}$ \\
\hline Lavender oil $0.50 \%$ & $0.30 \mathrm{a}$ & $0.24 \mathrm{a}$ & $0.20 \mathrm{a}$ & $0.15 \mathrm{a}$ & $0.26 \mathrm{a}$ & $0.24 \mathrm{a}$ & $0.20 \mathrm{a}$ & $0.17 \mathrm{a}$ \\
\hline Lemongrass oil $0.25 \%$ & $0.20 \mathrm{~d}$ & $0.18 \mathrm{c}$ & $0.11 \mathrm{~d}$ & $0.11 \mathrm{~cd}$ & $0.23 \mathrm{bc}$ & $0.19 b$ & $0.17 b$ & $0.13 \mathrm{c}$ \\
\hline Lemongrass oil $0.50 \%$ & $0.27 \mathrm{~b}$ & $0.24 \mathrm{a}$ & $0.19 a b$ & $0.13 \mathrm{bc}$ & $0.26 \mathrm{a}$ & $0.23 \mathrm{a}$ & $0.19 a b$ & $0.16 \mathrm{ab}$ \\
\hline Thyme oil $0.25 \%$ & $0.24 \mathrm{c}$ & $0.23 \mathrm{a}$ & $0.16 \mathrm{c}$ & $0.12 \mathrm{~cd}$ & $0.21 \mathrm{~cd}$ & $0.18 \mathrm{bc}$ & $0.13 d$ & -- \\
\hline Thyme oil & $0.25 \mathrm{bc}$ & $0.22 \mathrm{ab}$ & $0.15 c$ & $0.13 \mathrm{bc}$ & $0.20 \mathrm{~d}$ & $0.18 \mathrm{bc}$ & $0.16 \mathrm{c}$ & $0.14 \mathrm{bc}$ \\
\hline \multicolumn{9}{|c|}{2019 Season } \\
\hline Control & $0.24 \mathrm{c}$ & $0.19 \mathrm{~d}$ & $0.15 \mathrm{~d}$ & $0.10 \mathrm{c}$ & $0.22 \mathrm{~cd}$ & $0.17 c$ & $0.15 \mathrm{~b}$ & -- \\
\hline nder oil 0 & $0.28 \mathrm{~b}$ & $0.25 \mathrm{c}$ & $0.20 \mathrm{c}$ & $0.17 b$ & $0.24 \mathrm{bc}$ & $0.15 \mathrm{c}$ & $0.12 \mathrm{c}$ & $0.9 \mathrm{~d}$ \\
\hline Lavender oil $0.50 \%$ & $0.34 \mathrm{a}$ & $0.31 \mathrm{a}$ & $0.30 \mathrm{a}$ & $0.21 \mathrm{a}$ & $0.27 \mathrm{a}$ & $0.24 \mathrm{a}$ & $0.18 \mathrm{a}$ & $0.16 \mathrm{a}$ \\
\hline Lemongrass oil $0.25 \%$ & $0.30 \mathrm{~b}$ & $0.27 \mathrm{bc}$ & $0.25 \mathrm{~b}$ & $0.15 b$ & $0.22 \mathrm{~cd}$ & $0.20 \mathrm{~b}$ & $0.14 \mathrm{bc}$ & $0.11 \mathrm{~cd}$ \\
\hline Lemongrass oil $0.50 \%$ & $0.31 \mathrm{~b}$ & $0.29 \mathrm{~b}$ & $0.23 b$ & $0.15 b$ & $0.26 a b$ & $0.22 a b$ & $0.16 a b$ & $0.14 \mathrm{ab}$ \\
\hline Thyme oil $0.25 \%$ & $0.28 \mathrm{~b}$ & $0.25 c$ & $0.17 \mathrm{~d}$ & $0.10 \mathrm{c}$ & $0.21 \mathrm{~d}$ & $0.15 \mathrm{c}$ & $0.12 \mathrm{c}$ & -- \\
\hline Thyme oil $0.50 \%$ & $0.27 \mathrm{c}$ & $0.21 \mathrm{~d}$ & $0.16 \mathrm{~d}$ & $0.15 \mathrm{~b}$ & $0.24 \mathrm{bc}$ & $0.20 \mathrm{~b}$ & $0.16 a b$ & $0.13 \mathrm{bc}$ \\
\hline
\end{tabular}

Initial sample for recorded 0.31 and $0.36 \%$ for 2018 and 2019 seasons

Values followed by the same letter (s) are not significantly different at $5 \%$

\section{RECOMMENDATION}

From the obtained data it could be successfully storage of (Menthe piperita L.) fresh cut herb at $5^{\circ} \mathrm{C}$ for 15 days followed by 5 days at $20^{\circ} \mathrm{C}$ as imitation to marketing condition and the effect was more pronounced by treating the herb with lavender, lemongrass and thyme oils all at $0.50 \%$ before cold storage.

\section{REFERENCES}

A.O.A.C. 2005. Official Methods of the Analysis of AOAC. International18 ${ }^{\text {th }}$ Ed., Published by AOAC International. Maryland 20877-2417. USA.
Abd Elwahab W.A., Abd Elwahab S.M. and Kamel O.S. 2014. Using chitosan Ethanol, Bergamot oil, acetic acid and calcium chloride as safe alternatives to sulfur dioxide for control postharvest decay, maintain quality of crimson grape. M.Sc. Thesis Fac. of Agric. Cairo Univ. Egypt.

Abd-alla M.A., Abd-El-Kader M.M., Abd-ElKareem F. and El-Mohamedy R.S.R. 2011. Evaluation of lemongrass, thyme and peracetic acid against gray mold of strawberry fruits. Journal of Agricultural Technology, 7(6), 1775-1787. 
Almela, L., Sachez B., Fernandez J.A., Roca M. and Rabe V. 2006. Liquid chromatographic mass psectrometric analysis of phenolics and radical scavenging activity of rosemary extract from different raw material. J. Chromatography A, 1120: 221.

Aminifard M.H. and Mohammadi S. 2013. Essential oils to control Botrytis cinerea in vitro and in vivo on plum fruits. Journal of the Science of Food and Agriculture. 93(2), 348-353.

Arras G. and Usai M. 2001.Fungitoxic activity of twelve essential oils against four postharvest citrus pathogens: chemical analysis of Thymus capitatus (L.) oil and its effect in subatmospheric pressure conditions. J. Food Prot., 64, 10251029.

Bagamboula C.F., Uyttendaele M. and Debevere J. 2004. Inhibitory effect of thyme and basil essential oils, carvacrol, thymol, estragole, linalool and cymene towards Shigella sonnei and $S$. flexneri. Food Microbiol. 21, 33-42.

Bruneton J. 2005. Pharmacognosie, Phytochimie, Plantes medicinales, TEC \& DOC, Paris, 249250.

Camele I., De Feo V., Altier I.I., Mancini E., De Martino L. and Luiqi Rana G. 2010. An attempt of postharvest orange fruit rot control using essential oils from Mediterranean plants. J. Med. Food., 23, 45-52.

Charles D.J. and Simon J.E. 1990. Comparison of extraction methods for the rapid determination of essential oil content and composition of basil. J. Amer. Sochort Sci. 115(3), 458-462.

Combrinck S., Regnier T. and Kamatou G.P. 2011. In vitro activity of eighteen essential oils and some major components against common postharvest fungal pathogens of fruit. Ind. Crops Prod., 33(2), 344-349.

Dewanto V., Adom X.Z. and Liu K.K. 2002. Thermal processing enhances the nutritional value of tomatoes by increasing total antioxidant activity. J. Agricultural Food Chem. 50, 3010-3014.

Erkan M., Pekmezci M. and Wang C.Y. 2005. Hot water and curing treatments reduce chilling injury and maintain postharvest quality of 'Valencia' oranges. Int. J. of Food Sci. and Technology. 40, 91-96.

Fatemi S., Jafarpour M., Eghbalsaied S., Rezapour A. and Borji H. 2011. Effect of essential oils of Thymus vulgaris and Mentha piperita on the control of green mould and postharvestquality of Citrus Sinensis cv. Valencia. African J. of Biotechnology 10(66), 14932-14936.
Ghanbari M., Souri M.K., Mirzaei H.H. and Omidbaigi R. 2016. The essential oils composition in two populations of Achillea wilhelmsii C. Koch from Iran. Zeitschrift fur Arznei-und Gewurzpflanzen 21(3), 107-110.

Ghorbani A. and Esmaeilizadeh M. 2017. Pharmacological properties of Salvia officinalis and its components. Journal of Traditional and Complementary Medicine. 7(4), 433-440.

Heidarpour O., Souri M.K. and Omidbaigi R. 2013. Changes in content and constituents of essential oil in different plant parts of lovage ( $L e-$ visticum officinale Koch. Cv. Budakalaszi) cultivated in Iran. J. Essent Oil Bear Plants. 16(3), 318-322.

Hosoda K., Nishida T., Park W.Y. and Eruden B. 2005. Influence of eppermint (Mentha $\times$ piperita L.) supplementation on nutrient igestibility and energy metabolism in lactating dairy cows. Asian- Ustralian J. of Animal Sci., 18(12), 1721-1726.

Hyun J.E., Bae Y.M., Yoon J.H. and Lee S.Y. 2015. Preservative effectiveness of essential oils in vapor phase combined with modified atmosphere packaging against spoilage bacteria on fresh cabbage. Food Control 51, 307-313.

Isman M.B. and Machial C.M. 2006. Pesticides based on plant essential oils: from traditional practice to commercialization. Adv Phytomedicine; 3, 29-44.

Isman M.B. 2000. Plant essential oils for pest and disease management. Crop Prot., 19, 603-608.

Karkanis A, Lykas C., Liava V., Bezou A., Petropoulos S. and Tsiropoulos N. 2018. Weed interference with peppermint (Mentha $x$ piperita L. and spearmint (Mentha spicata L.) crops under different herbicid treatments: effects on biomass and essential oil yield. J. Sci., Food Agric., 98, 43-50.

Karthikesan K., Pari L. and Menon V.P. 2010. Antihyperlipidemic effect of chlorogenic acid and tetrahydrocurcumin in rats subjected to diabetogenic agents. Chemico-Biological Interactions; 188(3), 643-650.

Kushal G., Gurung S., Pokh S, Karn R. and Prakash A. 2020. Effect of different plant extracts on sprouting, storability and postharvest loss of potato In Baglung district, Nepal. Malaysian J. of Sustainable Agric., 4(1), 09-13.

Lalitha V., Kiran B. and Ravesha K.A. 2011. Antifungal and antibacterial potentiality of six essential oils extracted from plant source. Int. J. Eng. Sci. Technol. 3, 1029-3038. 
Lee Y., Kim J., Shin S., Lee S. and Park I. 2008. Antifungal activity of Myrtaceae essential oils and their components against three phytopathogenic fungi. Flavour Fragr. J., 23, 23-28.

Mahanta J.J., Chutia M., Bordoloi M., Pathak M.G., Adhikary R.K. and Sarma T.C. 2007. Cymbopogon citratus L. essential oil as a potential antifungal agent against key weed moulds of Pleurotus spp. Spawns. Flavour and Fragrance J., 22(6), 525-530.

Maisuthisakul P., Suttajit M. and Pongsawatmanit R. 2007 Assessment of phenolic content and free radical-scavenging capacity of some Thai indigenous plants. Food Chem. 100, 14091418.

Mari M., Bautista-Baños S. and Sivakumar D. 2016. Decay control in the postharvest system: role of microbial and plant volatile organic compounds. Postharvest Biol Technol., 122, 7081.

Mirdehghan S.H., Rahemi M., Martnez-Romero D., Valverde J.M., Zapata P.J., Serrano M. and Valero D. 2007. Reduction of pomegranate chilling injury during storage after heat treatment: role of polyamines. Postharvest Biol. Technol. 44, 19-25.

Mishra A.K. and Dubey N.K. 1994. Evaluation of some essential oils for their toxicity against fungi causing deterioration of stored food commodities. Appl. Environ. Microbiol 60(4), 11011105.

Mpho M., Sivakumar D., Selvam P., Sellamuthu A. and Bautista-Baños A.S. 2013. Use of lemongrass oil and modified atmosphere packing on control of anthrancnose and quality maintenace in avocado cultivars. J. of Food Quality pp. 198-208.

Nagata M. and Yamashta I. 1992. Simple method for simultaneous determination of chlorophyll and carotenoids in tomato fruits. J. Japan. Soc. Food Sci. Technol., 39(10), 925-928.
Novak J., Jan L., Friedrich P. and Chlodwig M.F. 2002. Essential oil compounds in a historical sample of marjoram (Origanum majorana L., Lamiaceae). Flavour and Fragrance J. 17, 175180.

Olivas G.I. and Barbosa-Cánovas G.V. 2005. Edible coatings for fresh-cut fruits. Critical Reviews in Food Sci. and Nutrition 45(7-8), 657670.

Plaza P., Torres R., Usall J., Lamarca N. and Vinas I. 2004. Evaluation of the potential of commercial post-harvest application of essential oils to control citrus decay. J. Hortic. Sci. Biotechnol. 79(6), 935-940.

Sandhya S.M. 2010. Modified atmosphere packaging of fresh produce: Current status and future needs. LWT-Food Sci. and Technology, 43(3), 381-392.

Şerban E.S., Ionescu M., Matinca D., Maier S. and Boji M. 2011. Screening of the antibacterial and antifungal activity of eight volatile essential oils. Farmacia, 59(3), 440-446.

Singleton V. and Rossi J.R. 1965. Colorimetry of total phenolics with phosphomonolybdic_/ phosphotungstic acid reagents. Am. J. Enol. Vitic. 16, 144-158.

Steel R.G., Torrie J.H. and Dickey Q. 1997. Principles and Procedures of Statistics. A Biometrical approach $3^{\text {rd }}$ Ed. McGraw Hill Book Co. Inc. New York. USA

Wang C.Y. 2003. Maintaining postharvest quality of raspberries with naturalvolatile compounds. Int. J. of Food Microbiology. 38, 869-875.

Wills I.R., Mcglasson B., Graham D. and Joyce D. 2007. Postharvest: an introduction to the physiology \& handling of fruit, vegetables \& ornamentals. $4^{\text {th }}$ ed. Wallingford: New South Wales University Press, 227 p.

Yousefizad L., Fathi R. and Ghanbari F. 2015. Effectiveness of $\mathrm{CaCl}_{2}$, peppermint oil and salicylic acid treatments on shelf life extension of fresh mint during cold storage. J. of Food Processing and Preservation, 39, 2639-2646. 


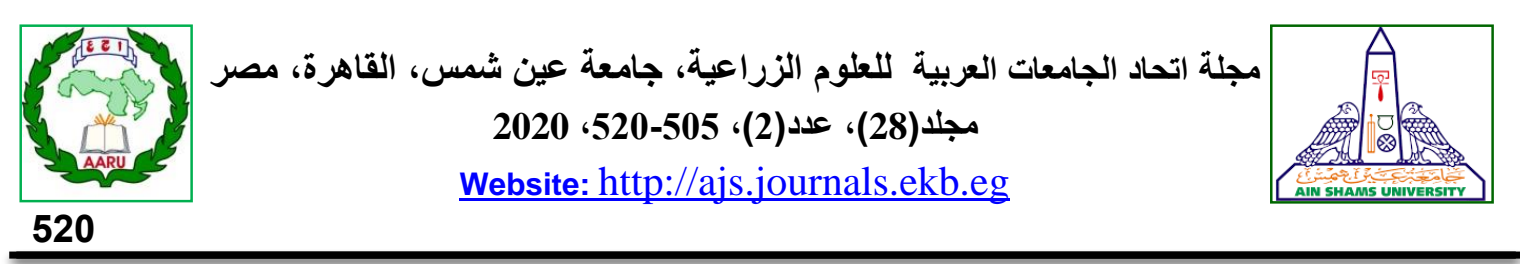

تأثير المعاملات ببعض الزيوت العطرية على جودة الحفظ للعشب الطازج لنبات النعناع تحت ظروف التخزين المبرد

[36]

\author{
أحمد نظمي عبد الحميد" \\ قسم البساتين - كلية الزراعة - جامعة عين شمس- ص.ب 68 - حدائق شبرا 11241 - القاهرة - مصر \\ *Corresponding author: nazmy604@yahoo.com
}

Received 13 July, 2020

Accepted 4 August, 2020

اشتملت علي الكلوروفيل الكلي- حمض الاسكوربيك

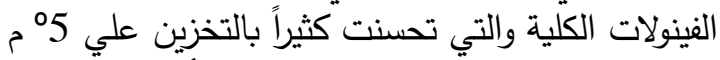

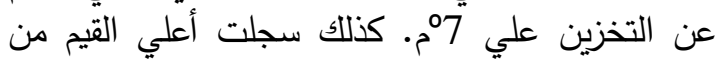

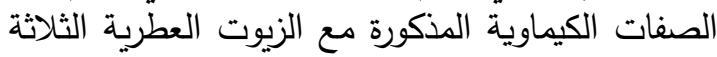

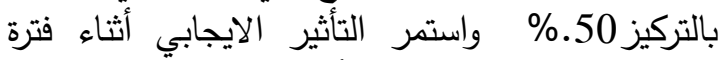

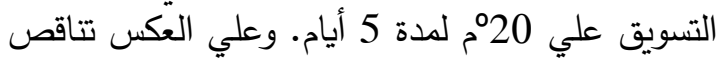

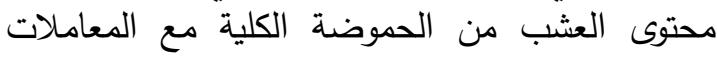
المختلفة مقارنة بالكنترول (ما عدا معاملة زيت الزيت الزعتر

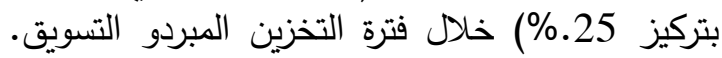
بالنسبة لصفة التسرب الآكتروليتي ومعدل التتفس فقدة الترن

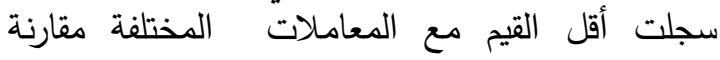

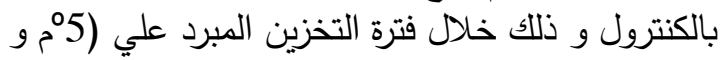

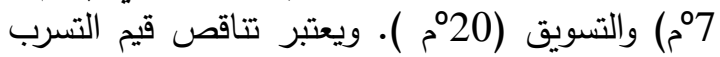

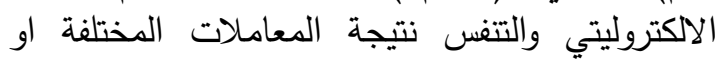

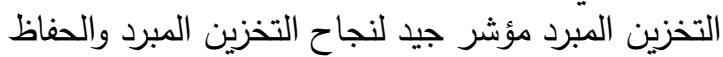

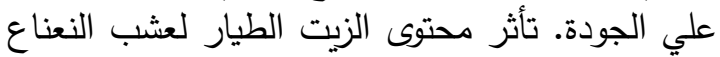

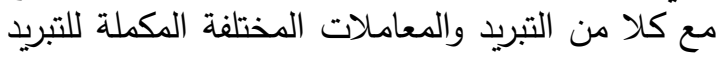

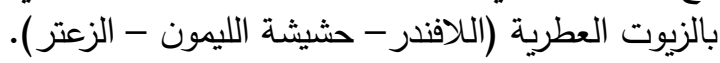

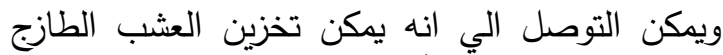

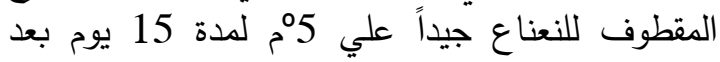

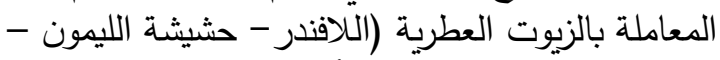
الزعتر) ثم التسويق لمدة 5 أيام علي 20 مالئم مع جودة

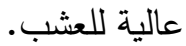

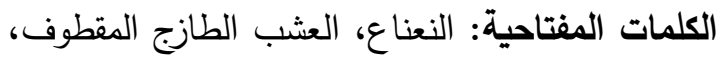

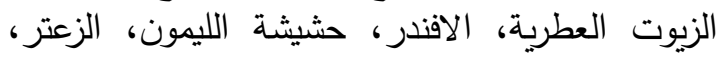

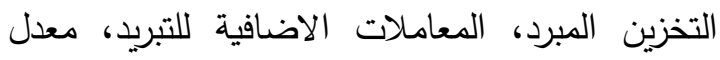
التتفس.

\section{الموجــز}

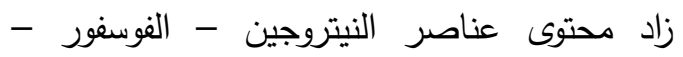

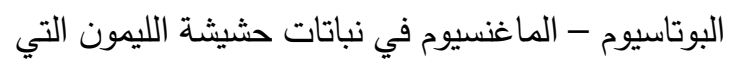

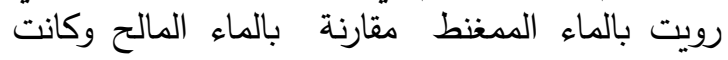

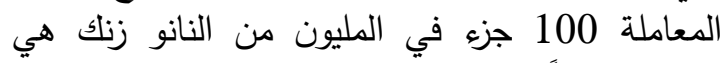

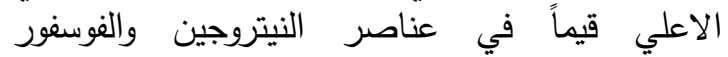

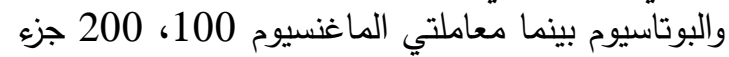

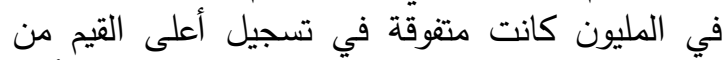

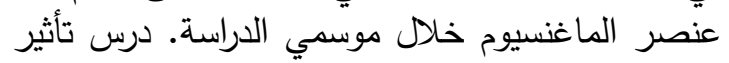

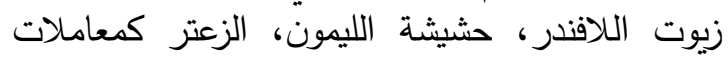

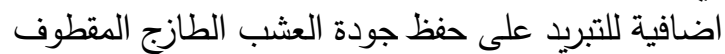

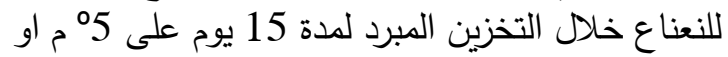

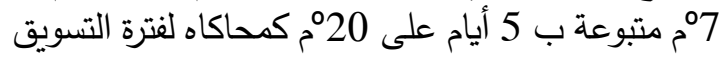

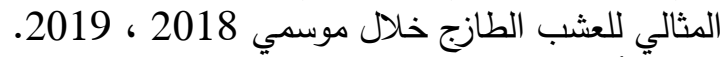
سجل تأثير ذللك علي الصفات الطبيعية - المكونات

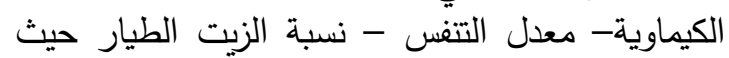

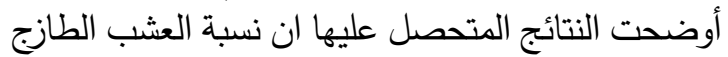

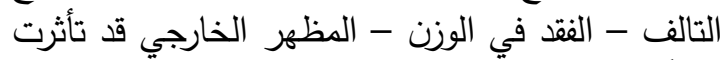
كثيراً بالمعاملات ألمختلفة وكان التأثير عند التخزين علي التئ تاني

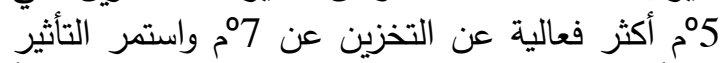

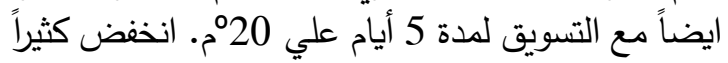
نسبة الفقد في الوزن ونسبة التالف مع التفائ المعاملات

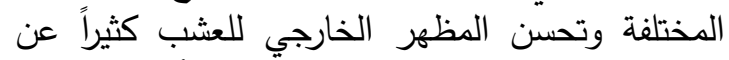
عشب المقارنة مع المعاملات المختلفة أثناء التخزين

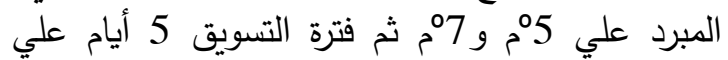
20م. استخدام الزيت العطري بتركيز 50. 50.

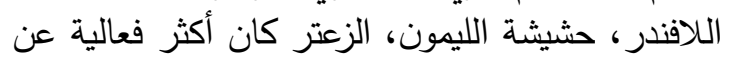
التركيز 25. من الزيوت الثلاثة. المكونات الكيماوية 